\title{
RELATIONS BETWEEN CONVOLUTIONS AND TRANSFORMS IN OPERATOR-VALUED FREE PROBABILITY
}

\author{
WEIHUA LIU
}

\begin{abstract}
We introduce a class of independence relations, which include free, Boolean and monotone independence, in operator valued probability. We show that this class of independence relations have a matricial extension property so that we can easily study their associated convolutions via Voiculescu's fully matricial function theory. Based the matricial extension property, we show that many results can be generalized to multivariable cases. Besides free, Boolean and monotone independence convolutions, we will focus on two important convolutions, which are orthogonal and subordination additive convolutions. We show that the operator-valued subordination functions, which come from the free additive convolutions or the operator-valued free convolution powers, are reciprocal Cauchy transforms of operator-valued random variables which are uniquely determined up to Voiculescu's fully matricial function theory. In the end, we study relations between certain convolutions and transforms in $C^{*}$-operator valued probability.
\end{abstract}

\section{INTRODUCTION}

1.1. Convolutions and transforms in $\mathbb{C}$-valued probability. Free probability is a theory introduced by Voiculescu for studying the reduced free products of operator algebras with specified states [31, 22]. For example, $C^{*}$-algebras with norm continuous states and Von Neumann algebras with normal states are frameworks for this theory. The elements from the algebras are called noncommutative random variables or random variables for short. A single selfadjoint random variable can be identified with a classical $\mathbb{R}$-valued random variable thus one can study its distribution. The notion of free independence is a key relation for random variables in free probability theory. Given two freely independent random variables $X$ and $Y$ whose distributions are $\mu$ and $\nu$, respectively, then the distribution of $X+Y$ is denoted by $\mu \boxplus \nu$, which is called the free additive convolution of $\mu$ and $\nu$. In the process of computing the distribution $\mu \boxplus \nu$, the Cauchy transform plays a central role. Given a probability measure $\mu$ on $\mathbb{R}$, then the Cauchy transform of $\mu$ is the function

$$
G_{\mu}(z)=\int_{\mathbb{R}} \frac{1}{z-t} d \mu(t): \mathbb{C}^{+} \rightarrow \mathbb{C}^{-}
$$


Voiculescu introduced the R-transform of $\mu$ to be

$$
R_{\mu}(z)=G_{\mu}^{\langle-1\rangle}(z)-\frac{1}{z},
$$

where $G_{\mu}^{\langle-1\rangle}$ is the inverse of $G_{\mu}$ with respect to composition. Voiculescu showed that

$$
R_{\mu \boxplus \nu}=R_{\mu}+R_{\mu},
$$

when $\mu$ and $\nu$ are compactly supported probability measures [32, 33]. Later, Bercovici and Voiculescu proved the relation (2) to probability measures with non-compact support [12]. Based on the linearization property (2) of the R-transform, one can define convolution powers with respect to $\boxplus$ such that if $\mu$ is a probability measure and $t>0$ is a real number, the convolution power $\mu^{\boxplus t}$ ( when it exists) is a probability measure uniquely determined by

$$
R_{\mu \boxplus t}=t R_{\mu}
$$

It is well known that $\mu^{\boxplus t}$ defines for all $t \geqslant 1$ and $\mu[22$.

In $\mathbb{C}$-valued noncommutative probability, there are independence relations other than free indenpendence, for example, Boolean independence [29], monotone independence [20], s-free independence [19] and orthogonal independence [19]. Similarly, we can define the Boolean additive convolution $\uplus$, the monotone additive convolution $\triangleright$, the s-free additive convolution $\boxplus$, the orthogonal additive convolution $\vdash$ for proability measures. Among these independence relations, Boolean independence is only commutative nonunital universal independence relation [27]. Given a probability measure $\mu$, we denote by $F_{\mu}$ the reciprocal Cauchy transform of $\mu$, that is

$$
F_{\mu}(z)=\frac{1}{G_{\mu}(z)}: \mathbb{C}^{+} \rightarrow \mathbb{C}^{+} .
$$

The Boolean additive convolution has the following property

$$
F_{\mu \uplus \nu}(z)=F_{\mu}(z)+F_{\nu}(z)-z
$$

where $\mu, \nu$ are probability measures and $\mu \uplus \nu$ is the Boolean convolution of $\mu$ and $\nu$ [16]. It is similar to the free additive convolutions, we have Boolean convolution powers $\mu^{\uplus t}$ such that if $t>0$ and $\mu$ is a probability measure, then $\mu^{\uplus^{t}}$ (always exists) is a probability uniquely determined by

$$
F_{\mu^{\uplus t}}(z)=t F_{\mu}(z)+(1-t) z .
$$

Monotone independence relation is a noncommutative relation but defines an associative convolutions. The monotone additive convolution has the following property

$$
F_{\mu \triangleright \nu}(z)=F_{\mu}\left(F_{\nu}(z)\right)
$$


where $\mu, \nu$ are probability measures and $\mu \triangleright \nu$ is the monotone convolution of $\mu$ and $\nu$ [16].

Orthogonal independence relation is introduced by Lenczewski [19] to study decompositions of the free additive convolutions. The additive convolution $\vdash$ associated with the orthogonal independence relation is neither commutative nor associative. The orthogonal additive convolution has the following analytic property

$$
F_{\mu \vdash \nu}(z)=F_{\mu}\left(F_{\nu}(z)\right)-F_{\nu}(z)+z
$$

where $\mu, \nu$ are probability measures and $\mu \vdash \nu$ is the orthogonal additive convolution of $\mu$ and $\nu$ [19]. From the relations (3), (4) and (5), one can easily see that

$$
\mu \triangleright \nu=\nu \uplus(\mu \vdash \nu) .
$$

The possibility for studying the free additive convolutions via Boolean and monotone additive convolutions is based on the fact that the Cauchy transform of the distribution $\mu \boxplus \nu$ is subordinated to the Cauchy transforms of $\mu$ and of $\nu$ on the upper half-plane $\mathbb{C}^{+}$[34]. That is

$$
F_{\mu \boxplus \nu}(z)=F_{\mu}\left(\omega_{1}(z)\right)=F_{\nu}\left(\omega_{2}(z)\right) .
$$

It is shown that $\omega_{1}(z)$ and $\omega_{2}(z)$ are reciprocal Cauchy transforms of certain distributions which are completely determined by $\mu$ and $\nu$ [14]. Therefore, subordination functions provide a new type of convolution, which is called subordination convolution or s-free additive convolution [19]. It is denoted by $\mu \boxminus \nu$ whose reciprocal Cauchy transform is $\omega_{1}(z)$ in Equation (7). In summary, we have the following property

$$
\left.F_{\mu \boxplus \nu}(z)=F_{\mu}\left(F_{\nu \boxplus \mu}(z)\right)=F_{\nu}\left(F_{\mu \boxplus \nu}(z)\right)=F_{\nu \boxplus \mu}(z)\right)+F_{\mu \boxplus \nu}(z)-z
$$

or

$$
\mu \boxplus \nu=\mu \triangleright(\nu \boxplus \mu)=\nu \triangleright(\mu \boxminus \nu)=(\nu \boxplus \mu) \uplus(\mu \boxminus \nu) .
$$

On should be careful that the s-free additive convolution $\boxminus$ is neither commutative nor associative. However, $\square$ is very powerful in studying free additive convolutions [3, 4, 10].

Let us brief review some relations between the convolutions we introduced before. In [7], Belinschi and Nica introduced a family $\left\{\mathbb{B}_{t} \mid t>0\right\}$ of transforms on probability measures by the formula

$$
\mathbb{B}_{t}(\mu)=\left(\mu^{\boxplus(1+t)}\right)^{\uplus \frac{1}{1+t}} .
$$

When $t=1$, the transform is a remarkable bijection discovered by Bercovici and Pata in their study of relations between $\boxplus$-infinite divisibility and $\uplus$-infinite divisibility [1]. Belinschi and Nica showed that these transforms form a semigroup with respect to composition, 
that is

$$
\mathbb{B}_{t}\left(\mathbb{B}_{s}(\mu)\right)=\mathbb{B}_{s+t}(\mu), \forall s, t>0 .
$$

Then, they showed that the relation (10) can be derived by the following relation between the free convolution powers and the Boolean convolution powers

$$
\left(\mu^{\boxplus p}\right)^{\uplus q}=\left(\mu^{\uplus q^{\prime}}\right)^{\boxplus p^{\prime}},
$$

where $p \geqslant 1, q>\frac{p-1}{p}, p^{\prime}=\frac{p q}{1-p+p q}, q^{\prime}=1-p+p q$ and $\mu$ is an algebraic probability distribution [8].

Let $\gamma$ be the free central limit law with variance 1 in free probability. In [8], Belinschi and Nica studied the free Brownian motion started at an arbitrary algebraic probability measure $\mu$ in the process $\left\{\mu \boxplus \gamma^{\boxplus t} \mid t \geqslant 0\right\}$. They defined a transform $\Phi$ for probability measures such that given a probability measure $\mu$ on $\mathbb{R}$, then $\Phi(\mu)$ is a probability measure on $\mathbb{R}$ such that

$$
F_{\Phi(\mu)}(z)=z-G_{\mu}(z) .
$$

Belinschi and Nica discovered that

$$
\Phi\left(\mu \boxplus \gamma^{\boxplus t}\right)=\mathbb{B}_{t}(\Phi)
$$

Later, Anshelevich generalized the relation (13) further by considering the free process $\left\{\mu \boxplus \nu^{\boxplus t} \mid t \geqslant 1\right\}$ where $\nu$ is an arbitrary probability measure [1].

Almost the above relations are interpreted via s-free additive convolutions which is fully developed in a combinatorial way by Nica [21]. First, Nica exhibited the following relation between the free convolutions and the s-free additive convolutions

$$
\left(\mu_{1} \boxplus \mu_{2}\right) \boxplus \nu=\left(\mu_{1} \boxplus \nu\right) \boxplus\left(\mu_{2} \boxplus \mu\right)
$$

and

$$
\left(\mu^{\boxplus t}\right) \boxplus \nu=(\mu \boxplus \nu)^{\boxplus t}
$$

where $\mu_{1}, \mu_{2}, \mu$ and $\nu$ are probability measures and $t$ is a positive real number. Nica also found the following relations between subordination convolutions and $\mathbb{B}$-transform, $\Phi$-transform

$$
\mu \boxminus \mu=\mathbb{B}_{1}(\mu)
$$

and

$$
\gamma \boxminus \mu=\mathbb{B}_{1}(\Phi(\mu))
$$

and

$$
\left.\mu^{\boxplus p}=\mu \triangleright\left(\mathbb{B}_{1}(\mu)\right)^{\boxplus p-1}\right) .
$$


Then, Anshelevich's free evolution with two states can be defined by the following formula

$$
\Phi[\mu, \nu]=\mathbb{B}_{1}^{-1}(\mu \boxminus \nu)
$$

and satisfies the following relation

$$
\Phi\left[\mu, \nu \boxplus \mu^{\boxplus t}\right]=\mathbb{B}_{t}(\Phi[\mu, \nu])
$$

or equivalently

$$
\mathbb{B}_{t}(\mu \boxminus \nu)=\mu \boxminus\left(\mu^{\boxplus t} \boxplus \nu\right)
$$

where $\mu, \nu$ are probability measures and $t \geqslant 1$.

It should be pointed out here that the results of Anshelevich, Belinschi and Nica are more general than what are listed above. In short, they considered $k$-tuples of random variables. In this paper, we are gonna show that $k$-tuples of random variables are special cases of operator valued random variables.

1.2. Operator valued probability. Soon after the birth of free probability, Voiculescu generalized the theory to an operator valued framework where the states in the original theory are replaced by bimodule maps [35. The initial analytic type of $R$-transform in operator valued free probability was first introduced by Voiculescu [35] and the combinatorial aspects of operator valued R-transforms were developed by Speicher [28]. Similarly, the operator valued R-transforms have the additive property (2). It follows that the convolution powers with respect to $\boxplus$ can be naturally generalized in operator-valued frame work if one uses positive real number power. Following a question due to Bercovici, in $C^{*}$-operator valued probability, the convolution powers with respect to $\boxplus$ was generalized further by Anshelevich, Belinschi, Fevrier, and Nica such that $t$ can be a completely positive map [2]. An explicit construction of the operator-valued free convolution powers was given by Shlyakhtenko [26]. Furthermore, a combinatrorial definition for the BelinschiNica's $\Phi$ is provided, then the relation (10) and the relation (13) are generalized in operator valued probability [2].

On the other hand, operator valued subordination functions were discovered Biane [13] and were reformulated by Voiculescu [37, 36] . As well as the scalar subordination functions, the subordination property is a very useful analytic property in operator-valued probability [6, 9, 5]. By studying the subordination property further, Voiculescu developed his fully matricial function theory [38, 39], from which the main tool in this paper is derived, for studying operator valued probability. A direct generalization of Cauchy transform is usually not enough to determine an operator-valued distribution, but this can be done by using Voiculescu's fully matricial operator valued functions. 
The goals of this paper are the following: In $C^{*}$-operator valued probability, we show that operator-valued subordination functions completely determine operator valued distributions in the fully matricial operator function theory. Therefore, we can define operatorvalued subordination convolutions or s-free additive convolutions. Furthermore, we show that Belinschi-Nica' s $\Phi$-transform is well defined in $C^{*}$-operator valued probability by using a fully matricial version of the equation (12). Then, we prove relations (11)-(21) in operator valued cases. We also show that many properties related to $k$-tuples of classical random variables can be reduced properties related to operator valued random variables.

Besides this introduction section, the rest of this paper is organized as follows: In Section 2, we introduce the main notions and tools to be used in this paper. In Section 3, we introduce a class of independence relations which are called $\mathcal{J}$-independence and their associated convolutions. We show a matricial property of the $\mathcal{J}$ independence relation, and explain that the $\mathcal{J}$-convolutions of $k$-tuples of $\mathcal{B}$-valued random variables can be decoded from the $\mathcal{J}$-convolutions of $\mathcal{B} \otimes M_{n}(\mathbb{C})$-valued random variables. In Section 4 , we study the reciprocal Cauchy transforms of Boolean convolutions. In Section 5, we study the reciprocal Cauchy transforms of Monotone convolutions. In Section 6, we introduce and study the operator valued orthogonal additive convolutions. In Section 7 , we introduce and study the operator valued s-free additive convolutions. After the preparations in the previous sections, in Section 8, we generalize the relations in Section 1.1 to the operator valued case.

\section{Preliminary}

2.1. B-valued Independence relations. We start with some necessary definitions in operator probability.

Definition 2.1. A $\mathcal{B}$-valued probability space $(\mathcal{A}, \mathbb{E}: \mathcal{A} \rightarrow \mathcal{B})$ consists of a unital algebra $\mathcal{B}$, a unital algebra $\mathcal{A}$ which is also a $\mathcal{B}-\mathcal{B}$ bimodule and a conditional expectation $\mathbb{E}$ : $\mathcal{A} \rightarrow \mathcal{B}$ i.e.

$$
\mathbb{E}\left[b_{1} a b_{2}\right]=b_{1} \mathbb{E}[a] b_{2}, \quad \mathbb{E}\left[b 1_{\mathcal{A}}\right]=b,
$$

for all $b_{1}, b_{2}, b \in \mathcal{B}, a \in \mathcal{A}$ and $1_{\mathcal{A}}$ is the unit of $\mathcal{A}$. The elements of $\mathcal{A}$ are called $\mathcal{B}$ valued random variables. Suppose that $\mathcal{B}$ is a unital $C^{*}$-algebra and $\mathcal{A}$ is a $*$-algebra, the conditional expectation $\mathbb{E}$ is said to be positive if

$$
\mathbb{E}\left[a a^{*}\right] \geqslant 0
$$

for all $a \in \mathcal{A}$. An element $x \in \mathcal{A}$ is selfadjoint if $x=x^{*}$.

We will denote by $(\mathcal{A}, \mathbb{E})$ short for $(\mathcal{A}, \mathbb{E}: \mathcal{A} \rightarrow \mathcal{B})$ when there is no confusion. Throughout this paper, we will assume that $\mathcal{A}$ and $\mathcal{B}$ are unital $C^{*}$-algebras. In this case, $(\mathcal{A}, \mathbb{E})$ is a called a $C^{*}$-operator valued probability space. 
Definition 2.2. We denote by $\mathcal{B}\langle X\rangle$ the algebra which is freely generated by $\mathcal{B}$ and the indeterminant $X$. The elements in $\mathcal{B}\langle X\rangle$ are called $\mathcal{B}$-polynomials. In addition, $\mathcal{B}\langle X\rangle_{0}$ denotes the subalgebra of $\mathcal{B}\langle X\rangle$ whose elements do not contain a constant term, i.e. the linear span of the noncommutative monomials $b_{0} X b_{1} X b_{2} \cdots b_{n-1} X b_{n}$, where $b_{k} \in \mathcal{B}$ and $n \geqslant 1$.

Definition 2.3. Given a a $\mathcal{B}$-valued probability space $(\mathcal{A}, \mathbb{E})$ and an element $a \in \mathcal{A}$, the distribution $\mu_{a}$ of $a$ is is a map from $\mathcal{B}\langle X\rangle$ to $\mathcal{B}$ such that

$$
\mu_{a}(P)=\mathbb{E}[P(a)]
$$

for all $P \in \mathcal{B}\langle X\rangle$. A $\mathcal{B}$-valued distribution $\mu$ is is a map from $\mathcal{B}\langle X\rangle$ to $\mathcal{B}$ such that $\mu=\mu_{a}$ from some $\mathcal{B}$-valued random variable $a$. If $a$ can be chose from $C^{*}$-operator $\mathcal{B}$ valued probability space, then $\mu$ is called a $\mathcal{B}$-valued distribution and will be denoted by $\mu \in \Sigma_{0}(\mathcal{B})$.

Definition 2.4. Given a $\mathcal{B}$-valued probability space $(\mathcal{A}, \mathbb{E})$.

- A family of unital $\mathcal{B}$ - $\mathcal{B}$-bimodule subalgebras $\left\{\mathcal{A}_{i}\right\}_{i \in I}$ of $\mathcal{A}$ are said to be freely independent with respect to $\mathbb{E}$ if

$$
\mathbb{E}\left[a_{1} \cdots a_{n}\right]=0
$$

whenever $i_{1} \neq i_{2} \neq \cdots \neq i_{n}, a_{k} \in \mathcal{A}_{i_{k}}$ and $\mathbb{E}\left[a_{k}\right]=0$ for all $k$. A family of $\left(x_{i}\right)_{i \in I}$ are said to be freely independent over $\mathcal{B}$, if the unital $\mathcal{B}$ - $\mathcal{B}$-bimodule subalgebras $\left\{\mathcal{A}_{i}\right\}_{i \in I}$ which are generated by $x_{i}$, respectively, are freely independent, or equivalently

$$
\mathbb{E}\left[p_{1}\left(x_{i_{1}}\right) p_{2}\left(x_{i_{2}}\right) \cdots p_{n}\left(x_{i_{n}}\right)\right]=0,
$$

whenever $i_{1} \neq i_{2} \neq \cdots \neq i_{n}, p_{1}, \ldots, p_{n} \in \mathcal{B}\langle X\rangle$ and $\mathbb{E}\left[p_{k}\left(x_{i_{k}}\right)\right]=0$ for all $k$.

- A family of non-unital $\mathcal{B}$ - $\mathcal{B}$-bimodule subalgebras $\left\{\mathcal{A}_{i}\right\}_{i \in I}$ of $\mathcal{A}$ are said to be boolean independent with respect to $\mathbb{E}$ if

$$
\mathbb{E}\left[a_{1} \cdots a_{n}\right]=\mathbb{E}\left[a_{1}\right] \mathbb{E}\left[a_{2}\right] \cdots \mathbb{E}\left[a_{n}\right]
$$

whenever $a_{k} \in \mathcal{A}_{i_{k}}$ and $i_{1} \neq i_{2} \neq \cdots \neq i_{n}$. A family of random variables $\left\{x_{i}\right\}_{i \in I}$ are said to be boolean independent over $\mathcal{B}$, if the non-unital $\mathcal{B}$ - $\mathcal{B}$-bimodule subalgebras $\left\{\mathcal{A}_{i}\right\}_{i \in I}$ which are generated by $x_{i}$ respectively are boolean independent, or equivalently

$$
\mathbb{E}\left[p_{1}\left(x_{i_{1}}\right) p_{2}\left(x_{i_{2}}\right) \cdots p_{n}\left(x_{i_{n}}\right)\right]=\mathbb{E}\left[p_{1}\left(x_{i_{1}}\right)\right] \mathbb{E}\left[p_{2}\left(x_{i_{2}}\right)\right] \cdots \mathbb{E}\left[p_{n}\left(x_{i_{n}}\right)\right],
$$

whenever $i_{1} \neq i_{2} \neq \cdots \neq i_{n}$ and $p_{1}, \ldots, p_{n} \in \mathcal{B}\langle X\rangle_{0}$. 
- If $\mathcal{I}$ is ordered,with order $>$. A family of non-unital $\mathcal{B}$ - $\mathcal{B}$-bimodule subalgebras $\left\{\mathcal{A}_{i}\right\}_{i \in I}$ of $\mathcal{A}$ are said to be monotone independent with respect to $E$ if

$$
\mathbb{E}\left[a_{1} \cdots a_{k-1} a_{k} a_{k+1} a_{n}\right]=\mathbb{E}\left[\left[a_{1} \cdots a_{k-1} \mathbb{E}\left[a_{k}\right] a_{k+1} a_{n}\right],\right.
$$

whenever $a_{k} \in \mathcal{A}_{i_{k}}$ and $i_{k}>i_{k-1}, i_{k+1}$. A family of random variables $\left\{x_{i}\right\}_{i \in I}$ are said to be boolean independent over $\mathcal{B}$, if the non-unital $\mathcal{B}$ - $\mathcal{B}$-bimodule subalgebras $\left\{\mathcal{A}_{i}\right\}_{i \in I}$ which are generated by $x_{i}$ respectively are monotone independent.

Definition 2.5. Let $\mu, \nu \in \Sigma_{0}(\mathcal{B})$ such that $\mu$ is equal to the distribution of $x$ and $\nu$ is the distribution of $y$, where $x$ and $y$ are freely(Boolean, Monotone) independent in a $\mathcal{B}$ valued probability space $(\mathcal{A}, \mathbb{E})$. Then the distribution of $x+y$ is called the free(Boolean, Monotone) additive convolution of $\mu$ and $\nu$, and will denoted by $\mu \boxplus \nu .(\mu \uplus \nu, \mu \triangleright \nu)$

2.2. Hilbert $C^{*}$-modules. Our constructions of $\mathcal{B}$-valued faimily of random variables of independence relations rely on the theory of Hilbert $C^{*}$-modules. We refer to [18] for a good introduction to this theory. If $\mathcal{B}=\mathbb{C}$, the theory becomes the classical Hilbert space theory.

Definition 2.6. An inner-product $\mathcal{B}$-module is a linear space $\mathcal{M}$ which is a $\mathcal{B}$-right module together with a map $(x, y) \rightarrow\langle x, y\rangle_{\mathcal{M}}: \mathcal{M} \otimes \mathcal{M} \rightarrow \mathcal{B}$ such that

1. $\langle x+y, z\rangle_{\mathcal{M}}=\langle x, z\rangle_{\mathcal{M}}+\langle y, z\rangle_{\mathcal{M}}$

2. $\langle x, y b\rangle_{\mathcal{M}}=\left(\langle x, y\rangle_{\mathcal{M}}\right) b$

3. $\langle x, y\rangle_{\mathcal{M}}=\langle y, x\rangle_{\mathcal{M}}^{*}$

4. $\langle x, x\rangle_{\mathcal{M}}>0$ if $x \neq 0$

for all $x, y, z \in \mathcal{M}$ and $b \in \mathcal{B}$.

$\mathcal{M}$ is a $\mathcal{B}-\mathcal{B}$ Hilbert bimodule if $\mathcal{M}$ is a $\mathcal{B}-\mathcal{B}$ bimodule and is complete with respect to the inner product $\langle,\rangle_{\mathcal{M}}$.

Definition 2.7. Let $\mathcal{M}$ be a $\mathcal{B}$ - $\mathcal{B}$ Hilbert bimodule. A map $t: \mathcal{M} \rightarrow \mathcal{M}$ is adjointable if there is a map $t^{*}: \mathcal{M} \rightarrow \mathcal{M}$ such that

$$
\langle t x, y\rangle_{\mathcal{M}}=\left\langle x, t^{*} y\right\rangle_{\mathcal{M}}
$$

for all $x, y \in M$. The set of all adjointable maps from $\mathcal{M}_{i}$ to $\mathcal{M}_{i}$ is denoted by $L(\mathcal{M})$.

It is well known that $L(\mathcal{M})$ is a $C^{*}$-algebra.

Definition 2.8. A $\mathcal{B}-\mathcal{B}$ Hilbert bimodule with a specified vector is a pair $(\mathcal{M}, \xi)$ consists of a $\mathcal{B}-\mathcal{B}$ Hilbert bimodule $\mathcal{M}$ and a vector $\xi \in \mathcal{M}$ such that $\langle\xi, b \xi\rangle_{\mathcal{M}}=b$ for $b \in \mathcal{B}$. $\mathcal{M}^{\circ}$ is the orthogonal completion of $\mathcal{B} \xi$. Then $\mathcal{M}=\mathcal{B} \xi \oplus \mathcal{M}^{\circ},\left(L(M),\langle\xi, \cdot \xi\rangle_{\mathcal{M}}\right)$ is a $C^{*}$-operator $\mathcal{B}$-valued probability space. 
Notation 2.9. We will denote $\langle\xi, \cdot \xi\rangle_{\mathcal{M}}$ by $\phi_{\xi}(\cdot)$.

Lemma 2.10. Let $(\mathcal{M}, \xi)$ be a $\mathcal{B}-\mathcal{B}$ Hilbert bimodule with a specified vector. Then

$$
b \xi=\xi b,
$$

for all $b \in \mathcal{B}$.

Proof. Since $\xi b \in \mathcal{B} \xi$, we have

$$
\left\langle b^{\prime} \xi+m, b \xi\right\rangle_{\mathcal{M}}=b^{\prime *} b=\left\langle b^{\prime} \xi+m, \xi\right\rangle_{\mathcal{M}} b=\left\langle b^{\prime} \xi+m, \xi b\right\rangle_{\mathcal{M}}
$$

for all $b^{\prime} \in \mathcal{B}$ and $m \in \mathcal{M}^{\circ}$. The proof is done.

Let $x \in L(\mathcal{M})$ be a selfadjoint operator. Then we can write

$$
x=\left(\begin{array}{cc}
p & a \\
a^{*} & T
\end{array}\right),
$$

where $a: \mathcal{B} \xi \rightarrow \mathcal{M}^{\circ}, T: \mathcal{M}^{\circ} \rightarrow \mathcal{M}^{\circ}, p: \mathcal{B} \xi \rightarrow \mathcal{B} \xi$. Notice that, $p$ can be identified as a map from $\mathcal{B}$ to $\mathcal{B}$, we have the following result.

Lemma 2.11. $p$ is a $\mathcal{B}$ constant, namely $p \in \mathcal{B}$.

Proof.

$$
p b=\langle\xi, p b \xi\rangle_{\mathcal{M}}=\langle\xi, p \xi b\rangle_{\mathcal{M}}=\langle\xi, p \xi\rangle_{\mathcal{M}} b,
$$

for all $b \in \mathcal{B}$.

Notation 2.12. We will denote the set $\left\{b \in \mathcal{B} \mid \frac{b-b^{*}}{i}>\epsilon 1_{\mathcal{B}}\right.$, for some $\left.\epsilon>0\right\}$ by $\mathbb{H}^{+}$and $\mathbb{H}^{-}=-\mathbb{H}^{+}$.

Definition 2.13. Given a distribution $\mu \in \Sigma_{0}(\mathcal{B})$ such that $\mu=\mu_{a}$, where $a$ is $\mathcal{B}$-valued random variable in $(\mathcal{A}, \mathbb{E})$. The $\mathcal{B}$-Cauchy transform $G_{\mu, 1}$ of $\mu$ is a map from $\mathbb{H}^{+} \rightarrow \mathbb{H}^{-}$ defined by

$$
G_{\mu, 1}(b)=\mathbb{E}\left[\left(b 1_{\mathcal{A}}-a\right)^{-1}\right],
$$

whereas $F_{\mu, 1}(b)=\left(G_{\mu, 1}(b)\right)^{-1}: \mathbb{H}^{+} \rightarrow \mathbb{H}^{+}$is called the $\mathcal{B}$-reciprocal Cauchy transform of $\mu$.

Given a distribution $\mu \in \Sigma_{0}(\mathcal{B})$, following the construction of Section 1.1 in [15], there exists a $\mathcal{B}-\mathcal{B}$ Hilbert bimodule with a specified vector $(\mathcal{M}, \xi)$ and a element $x \in L(\mathcal{M})$ such that $\mu$ is equal to the distribution $\mu_{x}$ of $x$ in $\left(L(\mathcal{M}), \phi_{\xi}\right)$.

The following proposition shows a relation between the reciprocal Cauchy transform and the vector-conditional expectation $\phi_{\xi}$. 
Proposition 2.14. Let $b \in \mathbb{H}^{+}$and $x$ be a random variable in $\left(L(\mathcal{M}), \phi_{\xi}\right)$ such that $x$ has the following decomposition

$$
x=\left(\begin{array}{cc}
p & a \\
a^{*} & T
\end{array}\right)
$$

Then

$$
F_{\mu_{x}, 1}(b)=b-p-a^{*}\left[\left.(b-T)^{-1}\right|_{\mathcal{M}^{\circ}}\right] a,
$$

where $\left[\left.(b-T)^{-1}\right|_{\mathcal{M}^{\circ}}\right]$ is the inverse of the restriction of $T-b$ to $\mathcal{M}^{\circ}$.

Proof. Notice that $p$ and $T$ are selfadjoint, hence $b-p$ is invertible in $\mathcal{B}$ and $b-T$ is invertible in $L\left(\mathcal{M}^{\circ}\right)$. With a direct computation, we have that

$$
\left(\begin{array}{cc}
b-p & a \\
a^{*} & b-T
\end{array}\right)^{-1}=\left(\begin{array}{cc}
\left(b-p-a^{*}\left[\left.(b-T)^{-1}\right|_{\mathcal{M}^{\circ}}\right] a\right)^{-1} & \ldots \\
\cdots & \ldots
\end{array}\right)
$$

By Lemma 2.11, we have that $\left(b-p-a^{*}\left[\left.(b-T)^{-1}\right|_{\mathcal{M}^{\circ}}\right] a\right)^{-1} \in \mathcal{B}$. Therefore, we have

$$
\begin{aligned}
F_{\mu_{x}, 1}(b) & =\phi_{\xi}\left((b-x)^{-1}\right)^{-1} \\
& =\left(\left(b-p-a^{*}\left[\left.(b-T)^{-1}\right|_{\mathcal{M}^{\circ}}\right] a\right)^{-1}\right)^{-1} \\
& =b-p-a^{*}\left[\left.(b-T)^{-1}\right|_{\mathcal{M}^{\circ}}\right] a .
\end{aligned}
$$

The proof is done.

2.3. Fully matricial functions. In this subsection, we introduce Voiculescu's fully matricial function theoery in $C^{*}$-algebra operator valued probility. We also refer to [17] for a good development of the theory.

Let $(\mathcal{A}, \mathbb{E})$ be a $C^{*}$-algebra operator valued probability space. A fully noncommutative $\mathcal{A}$-set $\Omega=\left(\Omega_{n}\right)_{n \geqslant 1}$ is a sequence such that $\Omega_{n} \in \mathcal{A} \otimes M_{n}(\mathbb{C})$ and $\Omega_{m+n} \cap\left(\mathcal{A} \otimes M_{n}(\mathbb{C}) \oplus\right.$ $\left.\mathcal{A} \otimes M_{m}(\mathbb{C})\right)=\Omega_{n} \oplus \Omega_{m}$ and $\left(1_{\mathcal{A}} \otimes S\right) \Omega_{n}\left(1_{\mathcal{A}} \otimes S^{-1}\right)=\Omega_{n}$ for all $n, S \in G L_{n}(\mathbb{C})$.

Definition 2.15. A fully matrial $\mathcal{B}$-valued function on a fully matricial $\mathcal{A}$-set $\Omega$ is a sequence $\left(f_{n}\right)_{n \geqslant 1}$ such that

1. $f_{n}: \Omega_{n} \rightarrow \mathcal{B} \otimes M_{n}(\mathbb{C})$ is a function for each $n$.

2. If $g^{\prime} \in \Omega_{m}$ and $g^{\prime \prime} \in \Omega_{n}$, then $f_{m+n}\left(g^{\prime} \oplus g^{\prime \prime}\right)=f_{n}\left(g^{\prime}\right) \oplus f_{m}\left(g^{\prime \prime}\right)$,

3. If $S \in G L_{n}(\mathbb{C})$ and $g \in \Omega_{n}$, then $f_{n}\left(\left(S \otimes 1_{\mathcal{A}}\right) g\left(S^{-1} \otimes 1_{\mathcal{A}}\right)\right)=\left(S \otimes 1_{\mathcal{A}}\right) f_{n}(g)\left(S^{-1} \otimes\right.$ $\left.1_{\mathcal{A}}\right)$.

Example 2.16. For each $n \geqslant 1$, let $\left(\mathcal{A} \otimes M_{n}(\mathbb{C}), \mathbb{E} \otimes I_{n}\right)$ be a $\mathcal{B} \otimes M_{n}(\mathbb{C})$-valued probability space such that

$$
\left(\mathbb{E} \otimes I_{n}\right)\left[\left(a_{i, j}\right)_{i, j=1, \ldots, n}\right]=\left(\mathbb{E}\left[a_{i, j}\right]\right)_{i, j=1, \ldots, n},
$$


and

$$
\mathbb{H}_{n}^{+}=\left\{b_{n} \in M_{n}(\mathcal{B}) \mid \frac{b_{n}-b_{n}^{*}}{i}>\epsilon 1_{n}, \text { for some } \epsilon>0\right\}
$$

and $a \in \mathcal{A}$ be a selfadjoint random variable. Let $\Omega_{n}=\left\{\left(a \otimes \mathcal{I}_{n}-b_{n}\right)^{-1} \mid b_{n} \in \mathbb{H}_{n}^{+}\right\}$. Then $\Omega=(\Omega)_{n \geqslant 1}$ is a fully noncommutative $\mathcal{A}$-set and $\left(\mathbb{E} \otimes I_{n}\right)_{n \geqslant 1}$ is a fully matricial function on $\Omega$.

With the help of the fully matricial function $\left(\mathbb{E} \otimes I_{n}\right)_{n \geqslant 1}$, we can define the following sequences of functions. Given a selfadjoint random variable $x \in \mathcal{A}$, let $\mu$ be the distribution of $a$. The matricial Cauchy transform of $\mu$ is a sequence of function $G_{\mu}=\left(G_{\mu, n}\right)_{n \geqslant 1}$ such that

$$
G_{\mu, n}\left(b_{n}\right)=\mathbb{E} \otimes I_{n}\left[\left(b_{n}-a \otimes I_{n}\right)^{-1}\right]: \mathbb{H}_{n}^{+} \rightarrow \mathbb{H}_{n}^{-}
$$

In addition, the sequence of functions $F_{\mu}=\left(F_{\mu, n}\right)_{n \geqslant 1}$, where

$$
F_{x, n}\left(b_{n}\right)=\left(G_{x, n}\left(b_{n}\right)\right)^{-1}: \mathbb{H}_{n}^{+} \rightarrow-\mathbb{H}_{n}^{+}
$$

is called the matricial reciprocal Cauchy transform of $\mu$. We will be using the following fact that illustrated in [5].

Proposition 2.17. Let $\mu, \nu \in \Sigma_{0}(\mathcal{B})$. Then $\mu=\nu$ if and only if $F_{\mu}=F_{\nu}$ on $\left(\mathbb{H}_{n}^{+}\right)_{n \geqslant 1}$.

The matricial R-transform $R=\left(R_{\mu, n}\right)_{n \geqslant 1}$ of $\mu \in \Sigma_{0}(\mathcal{B})$ is a sequence of functions defined as follows:

$$
R_{\mu, n}\left(b_{n}\right)=G_{\mu, n}^{\langle-1\rangle}\left(b_{n}\right)-b^{-1}
$$

where $G_{\mu, n}^{\langle-1\rangle}$ is the left inverse of $G_{\mu, n}$, namely $G_{\mu, n}^{\langle-1\rangle}\left(G_{\mu, n}\left(b_{n}\right)\right)=b_{n}$ for all $b_{n} \in \mathbb{H}_{n}^{+}$. In general, $R_{\mu, n}$ is not defined on $\mathbb{H}_{n}^{+}$, but in a uniform neighborhood of 0 . The matricial Voiculescu transform $\phi_{\mu}=\left(\phi_{\mu, n}\right)_{n \geqslant 1}$ is defined as $\phi_{\mu, n}(b)=R_{\mu, n}\left(b^{-1}\right)$. Then, we have

$$
\phi_{\mu, n}\left(b_{n}\right)=F_{\mu, n}^{\langle-1\rangle}\left(b_{n}\right)-b_{n},
$$

where $F_{\mu, n}^{\langle-1\rangle}$ is the left inverse of $F_{\mu, n}$. As the linearization property of the classical R-transform, we have

$$
R_{\mu \boxplus \nu, n}=R_{\mu, n}+R_{\nu, n}
$$

and

$$
\phi_{\mu \boxplus \nu, n}=\phi_{\mu, n}+\phi_{\nu, n}
$$

for all $n \geqslant 1$. 


\section{OPERATOR VALUED INDEPENDENCE RELATIONS AND THEIR MATRICIAL EXTENSIONS}

In this section, we introduce a class of independence relations include free, boolean and monotone independence.

Definition 3.1. Let $\mathcal{I}$ be an index set. We call

$$
\mathcal{K}(\mathcal{I})=\{\varnothing\} \amalg\left\{\left(i_{1}, \cdots, i_{n}\right) \in \mathcal{I}^{n} \mid i_{k} \neq i_{k+1} \text { for } k=1, \cdots, n-1, n \in \mathbb{N}\right\}
$$

the set of series associated with $\mathcal{I}$.

For each $i \in \mathcal{I}$, let $\left(\mathcal{M}_{i}, \xi_{i}\right)$ be a $\mathcal{B}$ - $\mathcal{B}$ bimodule with a specified vector $\xi_{i}$ such that $\left\langle b_{1} \xi_{i}, b_{2} \xi_{i}\right\rangle_{\mathcal{M}_{i}}=b_{1}^{*} b_{2}$ for all $b_{1}, b_{2} \in \mathcal{B}$, where $\langle\cdot, \cdot\rangle_{\mathcal{M}_{i}}$ is the inner product on $\mathcal{M}_{i}$. The reduced free product of $\left(\mathcal{M}_{i}, \xi_{i}\right)_{i \in \mathcal{I}}$ with amalgamation over $\mathcal{B}$ is given by

$$
\mathcal{M}=\mathcal{B} \xi \oplus \bigoplus_{n=1}^{\infty} \bigoplus_{i_{1} \neq i_{2} \neq \cdots \neq i_{n}} \mathcal{M}_{i_{1}}^{\circ} \otimes_{\mathcal{B}} \cdots \otimes_{\mathcal{B}} \mathcal{M}_{i_{n}}^{\circ} .
$$

where the inner product $\langle\cdot, \cdot\rangle_{\mathcal{M}}$ on $\mathcal{M}$ is recursively defined as follows:

$\left\langle m_{1} \otimes \cdots \otimes m_{p}, m_{1}^{\prime} \otimes \cdots \otimes m_{q}^{\prime}\right\rangle_{\mathcal{M}}=\delta_{p, q} \delta_{i_{1}, j_{1}}\left\langle m_{2} \otimes \cdots \otimes m_{p},\left\langle m_{1}, m_{1}^{\prime}\right\rangle_{\mathcal{M}_{i_{1}}} m_{2}^{\prime} \otimes \cdots \otimes m_{q}^{\prime}\right\rangle_{\mathcal{M}}$, where $m_{k} \in \mathcal{M}_{i_{k}}^{\circ}$ for $k=1, \cdots p$ and $m_{k}^{\prime} \in \mathcal{M}_{j_{k}}^{\circ}$ for $k=1, \ldots q .(\mathcal{M}, \xi)$ is a $\mathcal{B}$ - $\mathcal{B}$ bimodule with the specified vector $\xi$ such that $\left\langle b_{1} \xi, b_{2} \xi\right\rangle_{\mathcal{M}}=b_{1}^{*} b_{2}$

Definition 3.2. Given $\mathbf{i}=\left(i_{1}, \cdots, i_{m}\right) \in \mathcal{K}(\mathcal{I})$, the $\mathbf{i}$-projection $P_{\mathbf{i}}^{\mathcal{M}}$ is the orthogonal projection from $\mathcal{M}$ onto its subspace

$$
\mathcal{M}_{i_{1}}^{\circ} \otimes_{\mathcal{B}} \cdots \otimes_{\mathcal{B}} \mathcal{M}_{i_{m}}^{\circ}
$$

For convenience, $\mathbf{i}=\{\varnothing\}$ when $m=0$, and $P_{\varnothing}^{\mathcal{M}}$ is the orthogonal projection onto the subspace $\mathcal{B} \xi$.

Let $\mathcal{J} \subset \mathcal{K}(\mathcal{I})$ be a subset of $\mathcal{K}(\mathcal{I})$. We denote by

$$
P_{\mathcal{J}}=\sum_{\mathbf{i} \in \mathcal{J}} P_{\mathbf{i}}^{\mathcal{M}}
$$

For each $i \in \mathcal{I}$, let

$$
\mathcal{M}^{\circ}(i)=\bigoplus_{n=1}^{\infty} \bigoplus_{i \neq i_{1} \neq i_{2} \neq \cdots \neq i_{n}} \mathcal{M}_{i_{1}}^{\circ} \otimes_{\mathcal{B}} \cdots \otimes_{\mathcal{B}} \mathcal{M}_{i_{n}}^{\circ}
$$

and

$$
\mathcal{M}(i)=\mathcal{B} \xi \oplus \mathcal{M}^{\circ}(i)
$$


Then, for each $i$, let $V_{i}: \mathcal{M}_{i} \otimes_{\mathcal{B}} \mathcal{M}(i) \rightarrow \mathcal{M}$ be the unitary operator defined as

$$
\begin{array}{ll}
V_{i}: & \xi_{i} \otimes_{\mathcal{B}} \xi \rightarrow \xi \\
V_{i}: & \xi_{i} \otimes_{\mathcal{B}} m(i) \rightarrow m(i) \\
V_{i}: & m_{i} \otimes_{\mathcal{B}} \xi \rightarrow m_{i} \\
V_{i}: & m_{i} \otimes_{\mathcal{B}} m(i) \rightarrow m_{i} \otimes_{\mathcal{B}} m(i),
\end{array}
$$

where $m(i) \in \mathcal{M}^{\circ}(i), m_{i} \in \mathcal{M}_{i}^{\circ}$.

Let $\lambda_{i}: L\left(\mathcal{M}_{i}\right) \rightarrow L(\mathcal{M})$ be the $*$-homomorphism given by

$$
\lambda_{i}(a)=V_{i}\left(a \otimes 1_{\mathcal{M}(i)}\right) V_{i}^{-1},
$$

where $1_{\mathcal{M}(i)}$ is the identity map on $\mathcal{M}$. It is well-know that the family of $\left\{\lambda_{i}\left(L\left(\mathcal{M}_{i}\right)\right)\right\}_{i \in I}$ is freely independent over $\mathcal{B}$ with respect to $\phi_{\xi}(\cdot)=\langle\xi, \cdot \xi\rangle_{\mathcal{M}}$.

Definition 3.3. Let $\mathcal{J}=\left\{\mathcal{J}_{i} \subset \mathcal{K}(\mathcal{I})\right\}$ be a family of subsets of $\mathcal{K}(\mathcal{I})$. We say that $\mathcal{J}$ is $\mathcal{I}$-compatible if for each $i \in \mathcal{I}$ we have that $\left(i \neq i_{1}, \cdots, i_{m}\right) \in \mathcal{J}_{i}$ if and only if $\left(i, i_{1}, \cdots, i_{m}\right) \in J_{i}$.

Proposition 3.4. Let $\mathcal{J}=\left\{\mathcal{J}_{i} \subset \mathcal{K}(\mathcal{I})\right\}$ be a $\mathcal{I}$-compatible family of subsets of $\mathcal{K}(\mathcal{I})$. Then, for all $i \in \mathcal{I}, P_{\mathcal{J}_{i}}^{\mathcal{M}}$ is commuting with all elements in $\lambda_{i}\left(L\left(\mathcal{M}_{i}\right)\right)$.

Proof. Since the family $\mathcal{J}=\left\{\mathcal{J}_{i} \subset \mathcal{K}(\mathcal{I})\right\}$ is $\mathcal{I}$-compatible, for each $i \in \mathcal{I}$, we have that $P_{\mathcal{J}_{i}}^{\mathcal{M}}$ is the orthogonal projection onto the subspaces

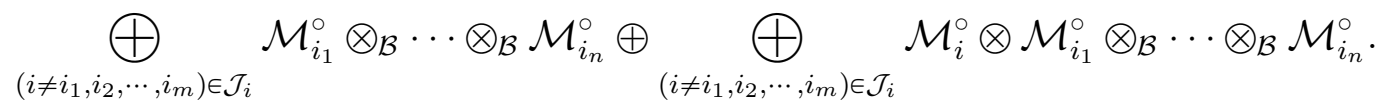

Let $\mathcal{J}_{i}^{\prime}=\left\{\left(i_{1}, i_{2}, \cdots, i_{m}\right) \in \mathcal{J}_{i} \mid i_{1} \neq i, m \in \mathbb{N}\right\}$. Then we have that

$$
\begin{aligned}
\lambda_{i}(a) P_{\mathcal{J}_{i}}^{\mathcal{M}} & =V_{i}\left(a \otimes 1_{\mathcal{M}(i)}\right) V_{i}^{-1} P_{\mathcal{J}_{i}}^{\mathcal{M}} \\
& =V_{i}\left(a \otimes 1_{\mathcal{M}(i)}\right)\left(1_{\mathcal{M}_{i}} \otimes P_{\mathcal{J}_{i}^{\prime}}^{\mathcal{M}}\right) V_{i}^{-1} \\
& =V_{i}\left(1_{\mathcal{M}_{i}} \otimes P_{\mathcal{J}_{i}^{\prime}}^{\mathcal{M}}\right)\left(a \otimes 1_{\mathcal{M}(i)}\right) V_{i}^{-1} \\
& =P_{\mathcal{J}_{i}}^{\mathcal{M}} V_{i}\left(a \otimes 1_{\mathcal{M}(i)}\right) V_{i}^{-1} .
\end{aligned}
$$

The proof is done.

By the commutativity of the projections defined by an $\mathcal{I}$-compatible family $\mathcal{J}$, we have the following result.

Corollary 3.5. Let $\mathcal{J}=\left\{\mathcal{J}_{i} \subset \mathcal{K}(\mathcal{I})\right\}$ be an $\mathcal{I}$-compatible family of subsets of $\mathcal{K}(\mathcal{I})$. Then, for each $i \in I, P_{\mathcal{J}_{i}} \lambda_{i}(\cdot)$ is a homomorphism from $L\left(\mathcal{M}_{i}\right)$ into $L(\mathcal{M})$.

Therefore, the following definition is well defined. 
Definition 3.6. Let $\left\{\mathcal{A}_{i} \mid i \in \mathcal{I}\right\}$ be a family of (necessarily unital) subalgebras of a $\mathcal{B}$ valued probability space $(\mathcal{A}, \mathbb{E})$. Given an $\mathcal{I}$-compatible family $\mathcal{J}=\left\{\mathcal{J}_{i} \subseteq \mathcal{K}\right\}$ of subsets of $\mathcal{K}$. We say that $\left\{\mathcal{A}_{i} \mid i \in \mathcal{I}\right\}$ are $\mathcal{B}$-valued $\mathcal{J}$-independent if there is a family $\left(\mathcal{M}_{i}, \xi_{i}\right)$ of $\mathcal{B}$ - $\mathcal{B}$ bimodules with specified vectors and $\mathcal{B}$-linear homomorphisms $\gamma_{i}: \mathcal{A}_{i} \rightarrow L\left(\mathcal{M}_{i}\right)$, on the reduced free product $(\mathcal{M}, \xi)=*_{i \in I}\left(\mathcal{M}_{i}, \xi_{i}\right)$, we have

$$
\mathbb{E}\left(a_{1} a_{2} \cdots a_{m}\right)=\phi_{\xi}\left(P_{\mathcal{J}_{i_{1}}}^{\mathcal{M}} \lambda_{i_{1}}\left(\gamma_{i_{1}}\left(a_{1}\right)\right) \cdots P_{\mathcal{J}_{i_{m}}}^{\mathcal{M}} \lambda_{i_{m}}\left(\gamma_{i_{m}}\left(a_{m}\right)\right)\right)
$$

whenever $a_{k} \in \mathcal{A}_{i_{k}}$ for $1 \leqslant k \leqslant m$.

Example 3.7. For each $i \in \mathcal{I}$, let $\mathcal{J}_{i}=\mathcal{K}(\mathcal{I})$. In this case, the $\mathcal{J}$-independence is the free independence.

Example 3.8. For each $i \in \mathcal{I}$, let $\mathcal{J}_{i}=\{\varnothing,(i)\}$. In this case, the $\mathcal{J}$-independence is the Boolean independence.

Example 3.9. Let $\mathcal{I}=\{1,2\}$ with the natural order, $J_{1}=\{\varnothing,(1)\}$ and $\mathcal{J}_{2}=\{\varnothing,(1),(2),(2,1)\}$. In this case, the $\mathcal{J}$-independence is the monotone independence.

Now, we show that the $\mathcal{J}$-independence relations have a matricial extension property on which allows us to apply the matricial functions.

Again, let $(\mathcal{A}, \mathbb{E})$ be a $\mathcal{B}$-valued probability space. Let $M_{n}(\mathbb{C})$ be the algebra of $n \times n$ matrices. For $1 \leqslant k, l \leqslant n$, let $e(k, l)$ be the element whose $(i, j)$-th entry is 1 and the other entries are 0 . Let $\mathbb{E}_{n}=\mathbb{E} \otimes I_{n}$ be the map from $\mathcal{A} \otimes M_{n}(\mathbb{C})$ to $\mathcal{B} \otimes M_{n}(\mathbb{C})$ defined as

$$
\mathbb{E}_{n}[a \otimes e(k, l)]=\mathbb{E}[a] \otimes e(k, l),
$$

whenever $a \in \mathcal{A}$ and $1 \leqslant k, l \leqslant n$.

Let $M_{i}=\mathcal{B} \xi_{i} \oplus \mathcal{M}_{i}^{\circ}$ be the $\mathcal{B}$ - $\mathcal{B}$ bimodule defined before. Then

$$
\mathcal{M}_{i} \otimes M_{n}(\mathbb{C})=\mathcal{B} \xi_{i} \otimes M_{n}(\mathbb{C}) \oplus \mathcal{M}_{i}^{\circ} \otimes M_{n}(\mathbb{C})
$$

is a $\mathcal{B} \otimes M_{n}(\mathbb{C})-\mathcal{B} \otimes M_{n}(\mathbb{C})$ bimodule with the specified vector $\xi_{i} \otimes I_{n}$ such that

$$
\left(b_{1} \otimes e\left(k_{1}, l_{1}\right)\right)(m \otimes e(k, l))\left(b_{2} \otimes e\left(k_{2}, l_{2}\right)\right)=\left(b_{1} m b_{2}\right) \otimes\left(e\left(k_{1}, l_{1}\right) e(k, l) e\left(k_{2}, l_{2}\right)\right),
$$

for $b_{1}, b_{2} \in \mathcal{B}, m \in \mathcal{M}_{i}$ and $1 \leqslant k_{1}, k_{2}, k, l_{1}, l_{2}, l \leqslant n$.

Notice that given two $\mathcal{B}-\mathcal{B}$ bimodules $\mathcal{M}_{1}$ and $\mathcal{M}_{2}$, we have that

$$
\left(\mathcal{M}_{1} \otimes M_{n}(\mathbb{C})\right) \otimes_{\mathcal{B} \otimes M_{n}(\mathbb{C})}\left(\mathcal{M}_{2} \otimes M_{n}(\mathbb{C})\right)=\left(\mathcal{M}_{1} \otimes_{\mathcal{B}} \mathcal{M}_{2}\right) \otimes_{\mathbb{C}} M_{n}(\mathbb{C}),
$$

where $\left(m_{1} \otimes e\left(k_{1}, l_{1}\right)\right) \otimes_{\mathcal{B} \otimes M_{n}(\mathbb{C})}\left(m_{2} \otimes e\left(k_{2}, l_{2}\right)\right)$ is identified with $\left(m_{1} \otimes_{\mathcal{B}} m_{2}\right) \otimes\left(e\left(k_{1}, l_{1}\right) e\left(k_{2}, l_{2}\right)\right)$. Therefore, the reduced free product of $\left(\mathcal{M}_{i} \otimes M_{n}(\mathbb{C}), \mathcal{B} \xi_{i} \otimes M_{n}(\mathbb{C})\right)_{i \in \mathcal{I}}$ is

$$
\mathcal{M} \otimes M_{n}(\mathbb{C})=\mathcal{B} \xi \otimes M_{n}(\mathbb{C}) \oplus \bigoplus_{n=1}^{\infty} \underset{i_{1} \neq i_{2} \neq \cdots \neq i_{n}}{\oplus} \mathcal{M}_{i_{1}}^{\circ} \otimes_{\mathcal{B}} \cdots \otimes_{\mathcal{B}} \mathcal{M}_{i_{n}}^{\circ} \otimes M_{n}(\mathbb{C})
$$


Let $\left(i_{1}, \cdots, i_{n}\right) \in \mathcal{K}(\mathcal{I})$. Then $P_{\left(i_{1}, \cdots, i_{n}\right)}^{\mathcal{M} \otimes M_{n}(\mathbb{C})}$ is the orthogonal projection from $\mathcal{M} \otimes M_{n}(\mathbb{C})$ onto the subspace

$$
\mathcal{M}_{i_{1}}^{\circ} \otimes_{\mathcal{B}} \cdots \otimes_{\mathcal{B}} \mathcal{M}_{i_{n}}^{\circ} \otimes M_{n}(\mathbb{C}) .
$$

Proposition 3.10. Let $\mathcal{I}$ be an index set. Given an $\mathcal{I}$-compatible family $\mathcal{J}=\left\{\mathcal{J}_{i} \subseteq\right.$ $\mathcal{K}(\mathcal{I}) \mid i \in \mathcal{I}\}$. Suppose that $\left\{\mathcal{A}_{i} \mid i \in \mathcal{I}\right\}$ is a $\mathcal{B}$-valued $\mathcal{J}$-independent family of subalgebras of a $\mathcal{B}$-valued probability space $(\mathcal{A}, \mathbb{E})$. Then, for each $n \in \mathbb{N},\left\{\mathcal{A}_{i} \otimes M_{n}(\mathbb{C}) \mid i \in \mathcal{I}\right\}$ a $\mathcal{B} \otimes$ $M_{n}(\mathbb{C})$-valued $\mathcal{J}$-independent family of subalgebras of the $\mathcal{B} \otimes M_{n}(\mathbb{C})$-valued probability space $\left(\mathcal{A} \otimes M_{n}(\mathbb{C}), \mathbb{E}_{n}\right)$.

Proof. Let $\mathbf{i}=\left(i_{1}, \cdots, i_{m}\right) \in \mathcal{K}$. Since $P_{\mathbf{i}}^{\mathcal{M} \otimes M_{n}(\mathbb{C})}$ is the orthogonal projection onto the subspace

$$
\mathcal{M}_{i_{1}}^{\circ} \otimes_{\mathcal{B}} \cdots \otimes_{\mathcal{B}} \mathcal{M}_{i_{m}}^{\circ} \otimes M_{n}(\mathbb{C})
$$

and $P_{\mathbf{i}}^{\mathcal{M}}$ is the orthogonal projection onto the subspace

$$
\mathcal{M}_{i_{1}}^{\circ} \otimes_{\mathcal{B}} \cdots \otimes_{\mathcal{B}} \mathcal{M}_{i_{m}}^{\circ}
$$

we have that $P_{\mathbf{i}}^{\mathcal{M} \otimes M_{n}(\mathbb{C})}=P_{\mathbf{i}}^{\mathcal{M}} \otimes 1_{M_{n}(\mathbb{C})}$.

For $1 \leqslant s \leqslant m$, let $\tilde{a_{s}}=a_{s} \otimes e\left(k_{s}, l_{s}\right) \in \mathcal{A}_{i_{s}} \otimes M_{n}(\mathbb{C})$, where $a_{s} \in \mathcal{A}_{i_{s}}$. We have

$$
\begin{aligned}
\mathbb{E}_{n}\left[\tilde{a_{1}} \cdots \widetilde{a_{m}}\right] & =\mathbb{E}\left[a_{1} \cdots a_{n}\right] \otimes\left(e\left(k_{1}, l_{1}\right) \cdots e\left(k_{m}, l_{m}\right)\right) \\
& =\phi_{\xi}\left(P_{\mathcal{J}_{i_{1}}} \lambda_{i_{1}}\left(\gamma_{i_{1}}\left(a_{1}\right)\right) \cdots P_{\mathcal{J}_{i_{n}}} \lambda_{i_{m}}\left(\gamma_{i_{m}}\left(a_{m}\right)\right)\right) \otimes\left(e\left(k_{1}, l_{1}\right) \cdots e\left(k_{m}, l_{m}\right)\right) \\
& =\left\langle\xi, P_{\mathcal{J}_{i_{1}}} \lambda_{i_{1}}\left(\gamma_{i_{1}}\left(a_{1}\right)\right) \cdots P_{\mathcal{J}_{i_{n}}} \lambda_{i_{m}}\left(\gamma_{i_{m}}\left(a_{m}\right)\right) \xi\right\rangle \otimes\left(e\left(k_{1}, l_{1}\right) \cdots e\left(k_{m}, l_{m}\right)\right) \\
& =\left\langle\xi \otimes I_{n}, P_{\mathcal{J}_{i_{1}}} \lambda_{i_{1}}\left(\gamma_{i_{1}}\left(a_{1}\right)\right) \otimes\left(e\left(k_{1}, l_{1}\right) \cdots P_{\mathcal{J}_{i_{n}}} \lambda_{i_{m}}\left(\gamma_{i_{m}}\left(a_{m}\right)\right) \otimes e\left(k_{m}, l_{m}\right)\right)\left(\xi \otimes I_{n}\right)\right\rangle \\
& =\left\langle\xi \otimes I_{n}, P_{\mathcal{J}_{i_{1}}}^{(n)} \lambda_{i_{1}, n}\left(\gamma_{i_{1}, n}\left(a_{1}\right) \otimes e\left(k_{1}, l_{1}\right) \cdots P_{\mathcal{J}_{i_{m}}}^{(n)} \lambda_{i_{m}}\left(\gamma_{i_{m}}\left(a_{m}\right) \otimes e\left(k_{m}, l_{m}\right)\right)\left(\xi \otimes I_{n}\right)\right\rangle\right. \\
& =\phi_{\xi \otimes I_{n}}\left(P _ { \mathcal { J } _ { i _ { 1 } } } ^ { ( n ) } \lambda _ { i _ { 1 } , n } \left(\gamma_{i_{1}, n}\left(a_{1}\right) \cdots P_{\mathcal{J}_{i_{m}}}^{(n)} \lambda_{i_{m}, n}\left(\gamma_{i_{m}, n}\left(a_{m}\right)\right) .\right.\right.
\end{aligned}
$$

By linearity of the previous map, for each $s, a_{s} \otimes e\left(k_{s}, l_{s}\right)$ can be replaced by an arbitrary element from $\mathcal{A}_{i_{s}}$. The proof is done.

3.1. $k$-tuples of operator valued random variables and convolutions. In this subsection, we assume that $k$ is a positive integer. Let $\mathcal{B}\left\langle X_{1}, \cdots, X_{k}\right\rangle$ be the set of noncommutative $\mathcal{B}$-valued polynomials in $k$ indeterminants, namely the linear span of $b_{0} X_{i_{1}} b_{1} X_{i_{2}} \cdots X_{i_{n}} b_{n}$ for $n \in \mathbb{N} \cup\{0\}, b_{0}, \cdots, b_{n} \in \mathcal{B}$ and $i_{1}, \cdots, i_{n} \in\{1, \cdots, k\}$.

Definition 3.11. Let $x_{1}, \cdots, x_{k}$ be random variables from a $\mathcal{B}$-valued probability space $(\mathcal{A}, \mathbb{E})$. The joint distribution $\mu$ of $x_{1}, \cdots, x_{k}$ is a $\mathcal{B}$-linear map from $\mathcal{B}\left\langle X_{1}, \cdots, X_{k}\right\rangle$ to $\mathcal{B}$ defined as follows

$$
\mu\left[b_{0} X_{i_{1}} b_{1} X_{i_{2}} \cdots X_{i_{n}} b_{n}\right]=\mathbb{E}\left[b_{0} x_{i_{1}} b_{1} x_{i_{2}} \cdots x_{i_{n}} b_{n}\right]
$$


Let $X=\sum_{l=1}^{k} x_{l} \otimes e(l, l)$. Then $X$ is a random variable in $\left(\mathcal{A} \otimes M_{n}(\mathbb{C}), \mathbb{E}_{n}\right)$. One can easily see that the $\mathcal{B} \otimes M_{n}(\mathbb{C})$-valued distribution of $X$ is completely determined by the joint distribution of $\left(x_{1}, \cdots, x_{k}\right)$. On the other hand, we have that

$$
\begin{aligned}
& \mathbb{E}\left[x_{i_{1}} b_{1} \cdots b_{m-1} x_{i_{m}}\right] \otimes e(1,1) \\
= & \left(\mathbb{E}_{n}\right)\left[\left(\left(1_{\mathcal{A}} \otimes e\left(1, i_{1}\right)\right) X\left(1_{\mathcal{A}} \otimes e\left(i_{1}, 1\right)\right)\right) b_{1} \otimes e(1,1) \cdots b_{m-1} \otimes e(1,1)\left(\left(1_{\mathcal{A}} \otimes e\left(1, i_{m}\right)\right) X\left(1_{\mathcal{A}} \otimes e\left(i_{m}, 1\right)\right)\right)\right] .
\end{aligned}
$$

Therefore, the joint distribution of $x_{1}, \cdots, x_{k}$ can be decoded from the $\mathcal{B} \otimes M_{n}(\mathbb{C})$ valued distribution of $X$. Further, recall that the free additive convolutions for $k$-tuples of random variables arise in the following way: Given joint distributions $\mu, \nu$ of $k$-tuples of random variables, we can always find random variables $x_{1}, \cdots, x_{k}, y_{1}, \cdots, y_{k}$ in a $\mathcal{B}$ valued probability space $(\mathcal{A}, \mathbb{E})$ such that $\mu$ is equal to the joint distribution of $x_{1}, \cdots, x_{k}$ and $\nu$ is equal to the joint distribution of $y_{1}, \cdots, y_{k}$, and the $\mathcal{B}$-algebra $\mathcal{A}_{1}$ generated by $x_{1}, \cdots, x_{k}$ is freely independent from the $\mathcal{B}$-algebra $\mathcal{A}_{2}$ generated by $y_{1}, \cdots, y_{k}$. Then, the free additive convolution $\mu \boxplus \nu$ is defined to be the joint distribution of $x_{1}+y_{1}, \cdots, x_{k}+y_{k}$. On the other hand, It is well know (or from Proposition $[3.10)$ that $\mathcal{A}_{1} \otimes M_{n}(\mathbb{C})$ is freely independent from $\mathcal{A}_{2} \otimes M_{n}(\mathbb{C})$ in the $\mathcal{B} \otimes M_{n}(\mathbb{C})$-valued probability $\left(\mathcal{A} \otimes M_{n}(\mathbb{C}), \mathbb{E}_{n}\right)$. It follows that $X=\sum_{l=1}^{k} x_{l} \otimes e(l, l)$ and $Y=\sum_{l=1}^{k} y_{l} \otimes e(l, l)$ are $\mathcal{B} \otimes M_{n}(\mathbb{C})$-freely independent in $\left(\mathcal{A} \otimes M_{n}(\mathbb{C}), \mathbb{E} \otimes I_{n}\right)$. Therefore, the free additive convolution $\mu \boxplus \nu$ of $\mu$ and $\nu$ can be decoded from the free additive convolution of the $\mathcal{B} \otimes M_{n}(\mathbb{C})$-valued distributions $\mu_{X}$ and $\nu_{Y}$, where $\mu_{X}$ is $\mathcal{B} \otimes M_{n}(\mathbb{C})$-valued distribution of $X$ and $\nu_{Y}$ is $\mathcal{B} \otimes M_{n}(\mathbb{C})$-valued distribution of $X$.

Now, we turn to introduce the additive convolutions associated with $\mathcal{J}$-independence. Even though the independence relations is well defined for subalgebras of a $\mathcal{B}$-valued probability, the $\mathcal{J}$-additive convolutions are not defined by $\mathcal{J}$-independence relations directly.

Let $\left\{\mu_{i}\right\}_{i \in I}$ be a family of $\mathcal{B}$-valued distributions, where $\mathcal{I}$ is a finite index set. Then, there are $\mathcal{B}$-valued operator valued probability spaces $\left(\mathcal{A}_{i}, \mathbb{E}_{i}\right)_{i \in \mathcal{I}}$ and $x_{i} \in \mathcal{A}_{i}$ such that for each $i, \mu_{i}$ is equal to the distribution of $x_{i}$. For each $i$, let $\left(\mathcal{M}_{i}, \xi_{i}\right)$ be a $\mathcal{B}-\mathcal{B}$ bimodule with a specified vector such that there exits a $\mathcal{B}$-linear homomorphism $\gamma_{i}: \mathcal{A}_{i} \rightarrow L\left(\mathcal{M}_{i}\right)$ and $\mathbb{E}_{i}[a]=\left\langle\xi_{i}, \gamma_{i}(a) \xi\right\rangle_{\mathcal{M}_{i}}$ for all $a \in \mathcal{A}_{i}$. Let $(\mathcal{M}, \xi)$ be the reduced free product of $\left(\mathcal{M}_{i}, \xi\right)$ and $\lambda_{i}: L\left(\mathcal{M}_{i}\right) \rightarrow L(M)$ are the embedding isomorphisms. Given an $\mathcal{I}$-compatible family $\mathcal{J}=\left\{\mathcal{J}_{i} \subset \mathcal{K}(\mathcal{I}) \mid i \in \mathcal{I}\right\}$, then

$$
X=\sum_{i \in \mathcal{I}} P_{\mathcal{J}_{i}}^{\mathcal{M}} \lambda_{i}\left(\gamma_{i}\left(x_{i}\right)\right) \in L(\mathcal{M}) .
$$


Then we can defined $\mu$ to be the distribution of $X$ in $(L(\mathcal{M}),\langle\xi, \cdot \xi\rangle)$. Now we show that $\mu$ is independent of the choices of $\left(\mathcal{M}_{i}, \xi_{i}\right)_{i \in \mathcal{I}}$. The proof follows Voiculescu's work in [40].

Definition 3.12. $\mathcal{B}$-morphisms between $\mathcal{B}$ - $\mathcal{B}$ bimodules with specified vector $\left(\mathcal{M}_{1}, \xi_{1}\right)$ and $\left(\mathcal{M}_{2}, \xi_{2}\right)$ are $\mathcal{B}$ - $\mathcal{B}$-linear maps $S: \mathcal{M} \rightarrow \mathcal{M}^{\prime}$, that is $S\left(b_{1} m b_{2}\right)=b_{1} S(m) b_{2}$, for all $m \in \mathcal{M}_{1}$ and $b_{1}, b_{2} \in \mathcal{B}$, such that

$$
S(\xi)=\xi^{\prime}
$$

and

$$
S\left(\mathcal{M}_{1}^{\circ}\right) \subseteq \mathcal{M}_{2}^{\circ} .
$$

Lemma 3.13. Let $(\mathcal{A}, \mathbb{E})$ be a $\mathcal{B}$-valued probability space. Let $\left(\mathcal{M}_{1}, \xi_{1}\right)$ and $\left(\mathcal{M}_{2}, \xi_{2}\right)$ be $\mathcal{B}-\mathcal{B}$ bimodules with specified vectors such that there exist homomorphisms $\gamma_{1}: \mathcal{A} \rightarrow$ $L\left(\mathcal{M}_{1}\right)$ and $\gamma_{2}: \mathcal{A} \rightarrow L\left(\mathcal{M}_{2}\right)$ such that

$$
\mathbb{E}[a]=\phi_{\xi_{1}}\left(\gamma_{1}(a)\right)=\phi_{\xi_{2}}\left(\gamma_{2}(a)\right),
$$

for all $a \in \mathcal{A}$. Then, there exists a $\mathcal{B}$-morphism $S: \mathcal{M}_{1} \rightarrow \mathcal{M}_{2}$ such that

$$
S \lambda_{1}(a)=\lambda_{2}(a) S
$$

for $a \in \mathcal{A}$.

Proof. Let $\mathcal{M}_{1}^{\prime}$ and $\mathcal{M}_{2}^{\prime}$ be the closure of the sub- $\mathcal{B}-\mathcal{B}$ bimodules generated by $\lambda_{1}(\mathcal{A}) \xi_{1}$ and $\lambda_{2}(\mathcal{A}) \xi_{2}$, respectively. Since $\mathbb{E}[a]=\phi_{\xi_{1}}\left(\gamma_{1}(a)\right)=\phi_{\xi_{2}}\left(\gamma_{2}(a)\right)$ for all $a \in \mathcal{A}$, the map $S_{0}: \lambda_{1}(\mathcal{A}) \xi_{1} \rightarrow \lambda_{2}(\mathcal{A}) \xi_{2}$ defined by $S_{0}\left(\lambda_{1}(a) \xi_{1}\right)=\lambda_{2}(a) \xi_{2}$ is an isomorphism such that $S_{0} \xi_{1}=\xi_{2}$. Let $S$ be the extension of $S_{0}$ to $\mathcal{M}_{1}$ such that the restriction $\left.S\right|_{\mathcal{M}_{1}^{\prime}{ }^{\perp}}=0$. Then $S$ is the $\mathcal{B}$-morphism we want.

Let $\left(\mathcal{M}_{i}^{\prime}, \xi_{i}^{\prime}\right)_{i \in I}$ be a family of $\mathcal{B}-\mathcal{B}$ bimodules with specified vectors such that for each $i$, there exits a $\mathcal{B}$-linear homomorphism $\gamma_{i}^{\prime}: \mathcal{A}_{i} \rightarrow L\left(\mathcal{M}_{i}^{\prime}\right)$ and $\mathbb{E}_{i}[a]=\phi_{\xi_{i}}(a)$ for all $a \in \mathcal{A}_{i}$. For each $i \in \mathcal{I}$, let $S_{i}$ be a $\mathcal{B}$-morphism from $\left(\mathcal{M}_{i}, \xi_{i}\right)$ to $\left(\mathcal{M}_{i}^{\prime}, \xi_{i}^{\prime}\right)$ such that

$$
S \lambda_{1}(a)=\lambda_{2}(a) S,
$$

for all $a \in \mathcal{A}$. Let $(\mathcal{M}, \xi)$ and $\left(\mathcal{M}^{\prime}, \xi^{\prime}\right)$ be the reduced free product of $\left(\mathcal{M}_{i}, \xi_{i}\right)_{i \in I}$ and $\left(\mathcal{M}_{i}^{\prime}, \xi_{i}^{\prime}\right)_{i \in I}$, respectively. Then, we have a $\mathcal{B}$-morphism $S:(\mathcal{M}, \xi) \rightarrow\left(\mathcal{M}^{\prime}, \xi^{\prime}\right)$ defined as

$$
S(b \xi)=b \xi^{\prime}, \quad \forall b \in \mathcal{B}
$$

and

$$
S\left(m_{1} \otimes_{\mathcal{B}} \cdots \otimes_{\mathcal{B}} m_{n}\right)=S_{i_{1}}\left(m_{1}\right) \otimes_{\mathcal{B}} \cdots \otimes S_{i_{n}}\left(m_{n}\right),
$$

for all $n$, where $m_{k} \in \mathcal{M}_{i_{k}}^{\circ}$ for $k=1, \cdots, n$. Let $\lambda_{i}^{\prime}$ be the embedding isomorphism from $L\left(M_{i}\right)$. It follows the proof of Lemma 1.13 in [40] that

$$
S \lambda_{i}\left(\gamma_{i}(a)\right)=\lambda_{i}^{\prime}\left(\gamma_{i}^{\prime}(a)\right) S .
$$


One can easily check that $S P_{\mathbf{i}}^{\mathcal{M}}=P_{\mathbf{i}}^{\mathcal{M}^{\prime}} S$ for all $\mathbf{i} \in \mathcal{K}(\mathcal{I})$. It follows that $S P_{\mathcal{J}_{i}}^{\mathcal{M}}=P_{\mathcal{J}_{i}}^{\mathcal{M}^{\prime}} S$ for all $i \in \mathcal{I}$. Therefore, we have that

$$
\begin{aligned}
& S P_{\mathcal{J}_{i_{1}}}^{\mathcal{M}} \lambda_{i_{1}}\left(\gamma_{i_{1}}\left(a_{1}\right)\right) \cdots P_{\mathcal{J}_{i_{m}}}^{\mathcal{M}} \lambda_{i_{m}}\left(\gamma_{i_{m}}\left(a_{m}\right)\right) \xi \\
= & P_{\mathcal{J}_{i_{1}}}^{\mathcal{M}^{\prime}} \lambda_{i_{1}}^{\prime}\left(\gamma_{i_{1}}^{\prime}\left(a_{1}\right)\right) \cdots P_{\mathcal{J}_{i_{m}}}^{\mathcal{M}^{\prime}} \lambda_{i_{m}}^{\prime}\left(\gamma_{i_{m}}^{\prime}\left(a_{m}\right)\right) \xi^{\prime} .
\end{aligned}
$$

According to the definition of $S$, we have that

$$
\phi_{\xi}\left[P_{\mathcal{J}_{i_{1}}}^{\mathcal{M}} \lambda_{i_{1}}\left(\gamma_{i_{1}}\left(a_{1}\right)\right) \cdots P_{\mathcal{J}_{i_{m}}}^{\mathcal{M}} \lambda_{i_{m}}\left(\gamma_{i_{m}}\left(a_{m}\right)\right)\right]=\phi_{\xi^{\prime}}\left[P_{\mathcal{J}_{i_{1}}}^{\mathcal{M}^{\prime}} \lambda_{i_{1}}^{\prime}\left(\gamma_{i_{1}}^{\prime}\left(a_{1}\right)\right) \cdots P_{\mathcal{J}_{i_{m}}}^{\mathcal{M}^{\prime}} \lambda_{i_{m}}^{\prime}\left(\gamma_{i_{m}}^{\prime}\left(a_{m}\right)\right)\right]
$$

whenever $a_{k} \in \mathcal{A}_{i_{k}}$ for $1 \leqslant k \leqslant m$. Therefore, $\left\{P_{\mathcal{J}_{i}}^{\mathcal{M}} \lambda_{i}\left(\gamma_{i}\left(x_{i}\right)\right) \mid i \in \mathcal{I}\right\}$ and $\left\{P_{\mathcal{J}_{i}^{\prime}}^{\mathcal{M}^{\prime}} \lambda_{i}^{\prime}\left(\gamma_{i}^{\prime}\left(x_{i}\right)\right) \mid i \in\right.$ $\mathcal{I}$ \} have the same joint distribution. It follows that the distribution of $X^{\prime}=\sum_{i \in \mathcal{I}} P_{\mathcal{J}_{i}}^{\mathcal{M}^{\prime}} \lambda_{i}^{\prime}\left(\gamma_{i}^{\prime}\left(x_{i}\right)\right)$ is also $\mu$. We have the following well-defined definition.

Definition 3.14. Given a finite index set $\mathcal{I}$, let $\left\{\mu_{i}\right\}_{i \in I}$ be a family of $\mathcal{B}$-valued distribution of $k$-tuples of random variables and $\mathcal{J}=\left\{\mathcal{J}_{i} \subset \mathcal{K}(\mathcal{I}) \mid i \in \mathcal{I}\right\}$ is $\mathcal{I}$-compatible. For each $i$, let $\left(\mathcal{M}_{i}, \xi_{i}\right)$ be a $\mathcal{B}$ - $\mathcal{B}$ bimodule with a specified vector and $x(i, 1), \cdots, x(i, k) \in \mathcal{M}_{i}$ such that $\mu_{i}$ is equal to the distribution of $x(i, 1), \cdots, x(i, k)$. Let $(\mathcal{M}, \xi)$ be the reduced free product of $\left(\mathcal{M}_{i}, \xi\right)$ and $\lambda_{i}: L\left(\mathcal{M}_{i}\right) \rightarrow L(M)$ are the embedding isomorphisms. Then, the $\mathcal{J}$-additive convolution of $\left\{\mu_{i}\right\}_{i \in I}$ is the distribution of $\left(X_{1}, \cdots, X_{k}\right)$, where

$$
X_{l}=\sum_{i \in \mathcal{I}} P_{\mathcal{J}_{i}}^{\mathcal{M}} \lambda_{i}(x(i, l))
$$

for $1 \leqslant l \leqslant k$.

Example 3.15. Let $\mathcal{I}=\{1,2\}$ and $\mathcal{J}=\left\{\mathcal{J}_{1}, \mathcal{J}_{2}\right\}$ such that $\mathcal{J}_{1}=\mathcal{J}_{2}=\mathcal{K}(\mathcal{I})$. Then the $\mathcal{J}$ additive convolution is the free additive convolution, namely the $\mathcal{J}$-additive convolution of $\mu_{1}$ and $\mu_{2}$ is $\mu_{1} \boxplus \mu_{2}$.

Example 3.16. Let $\mathcal{I}=\{1,2\}$ and $\mathcal{J}=\left\{\mathcal{J}_{1}, \mathcal{J}_{2}\right\}$ such that $\mathcal{J}_{1}=\{\varnothing,(1)\}$ and $\mathcal{J}_{2}=$ $\{\varnothing,(2)\}$. Then the $\mathcal{J}$-additive convolution is the Boolean additive convolution, namely the $\mathcal{J}$-additive convolution of $\mu_{1}$ and $\mu_{2}$ is $\mu_{1} \uplus \mu_{2}$.

Example 3.17. Let $\mathcal{I}=\{1,2\}$ and $\mathcal{J}=\left\{\mathcal{J}_{1}, \mathcal{J}_{2}\right\}$ such that $\mathcal{J}_{1}=\{\varnothing,(1)\}$ and $\mathcal{J}_{2}=$ $\{\varnothing,(1),(2),(2,1)\}$. Then the $\mathcal{J}$-additive convolution is the monotone additive convolution, namely the $\mathcal{J}$-additive convolution of $\mu_{1}$ and $\mu_{2}$ is $\mu_{1} \triangleright \mu_{2}$.

In this paper, we will study the following two $\mathcal{J}$-additive convolutions later.

Definition 3.18. Let $\mathcal{I}=\{1,2\}$ and $\mathcal{J}=\left\{\mathcal{J}_{1}, \mathcal{J}_{2}\right\}$ such that $\mathcal{J}_{1}=\{\varnothing,(1)\}$ and $\mathcal{J}_{2}=$ $\{(1),(2,1)\}$. Then the $\mathcal{J}$-additive convolution is called orthogonal additive convolution. The orthogonal additive convolution of $\mu_{1}$ and $\mu_{2}$ is denoted by $\mu_{1} \vdash \mu_{2}$. 
Definition 3.19. Let $\mathcal{I}=\{1,2\}$ and $\mathcal{J}=\left\{\mathcal{J}_{1}, \mathcal{J}_{2}\right\}$ such that $\mathcal{J}_{1}=\left\{\left(i_{1}, \cdots, i_{m}\right) \in\right.$ $\left.\mathcal{K}(\mathcal{I})) \mid i_{m}=1\right\} \cup\{\varnothing\}$ and $\left.\mathcal{J}_{2}=\left\{\left(i_{1}, \cdots, i_{m}\right) \in \mathcal{K}(\mathcal{I})\right) \mid i_{m}=1\right\}$. Then $\mathcal{J}=\left\{\mathcal{J}_{1}, \mathcal{J}_{2}\right\}$ is $\mathcal{I}$-compatible and the $\mathcal{J}$-additive convolution is called s-free additive convolution. The s-free additive convolution of $\mu_{1}$ and $\mu_{2}$ is denoted by $\mu_{1} \boxplus \mu_{2}$.

Remark 3.20. The orthogonal additive convolution and the s-free additive convolution are neither commutative nor associative.

Definition 3.21. Let $\left(x_{1}, \cdots, x_{k}\right)$ be a $k$-tuple of random variables in a $\mathcal{B}$-valued probability space $(\mathcal{A}, \mathbb{E})$. Then the matricial distribution of $\left(x_{1}, \cdots, x_{k}\right)$ is the $\mathcal{B} \otimes M_{n}(\mathbb{C})$-valued

distribution $\mu_{X}$ of $X=\sum_{l=1}^{k} x_{l} \otimes e(l, l) \in \mathcal{A} \otimes M_{n}(\mathbb{C})$ in the $\mathcal{B} \otimes M_{n}(\mathbb{C})$-valued probability $\left(\mathcal{A} \otimes M_{n}(\mathbb{C}), \mathbb{E} \otimes I_{n}\right)$.

According the proof of Proposition 3.10, we have the following property.

Proposition 3.22. Let $\left\{\mu_{i}\right\}_{i \in I}$ be a family of $\mathcal{B}$-valued distributions of $k$-tuples of random variables $(x(i, 1), \cdots, x(i, k)) \in\left(L\left(\mathcal{M}_{i}\right), \phi_{\xi_{i}}\right)$ for each $i$ and $\mu$ is a $\mathcal{B}$-valued distribution of a $k$-tuple of random variables $\left(x_{1}, \cdots, x_{k}\right) \in\left(L(\mathcal{M}), \phi_{\xi}\right)$. Then the $\mathcal{J}$-additive convolution of $\left\{\mu_{i}\right\}_{i \in I}$ is equal to $\mu$ if and only if the $\mathcal{J}$-additive convolution of the matricial distributions of $(x(i, 1), \cdots, x(i, k)) \in\left(L\left(\mathcal{M}_{i}\right), \phi_{\xi_{i}}\right)$ is equal to the matricial distribution of $\left(x_{1}, \cdots, x_{k}\right)$.

\section{On the Boolean additive Convolution and operator-Valued Boolean CONVOLUTION POWERS}

In this section, we study some analytic properties of the $\mathcal{B}$-valued Boolean additive convolution and the $\mathcal{B}$-valued Boolean convolution powers. The combinatorial aspects of $\mathcal{B}$-valued Boolean convolution are studied in [24].

\subsection{Boolean additive convolution.}

Lemma 4.1. Let $x, y$ be Boolean independent selfadjoint random variables from $(\mathcal{A}, \mathbb{E})$ and letalg $\{\mathcal{B}, x, y\}$ be is the algebra generated by $\mathcal{B}, x, y$. Then

$$
\mathbb{E}[a x f(y)]=\mathbb{E}[a x] \mathbb{E}[f(y)],
$$

for all $a \in \operatorname{alg}\{\mathcal{B}, x, y\}$ and $f \in \mathcal{B}\langle X\rangle$.

Proof. Notice that $a x$ is a linear combination of mononials ends with $x$, thus

$$
\mathbb{E}\left[a x f_{0}(y)\right]=\mathbb{E}[a x] \mathbb{E}\left[f_{0}(y)\right],
$$

for all $f_{0} \in \mathcal{B}_{0}\langle X\rangle$. 
Suppose that $b \in \mathcal{B}$ is the constant term of $f$, namely $f-b \in \mathcal{B}_{0}\langle X\rangle$. Then we have

$$
\mathbb{E}[a x(f(y)-b)+a x b]=\mathbb{E}[a x] \mathbb{E}[f(y)-b]+\mathbb{E}[a x] b=\mathbb{E}[a x] \mathbb{E}[f(y)]
$$

The proof is done.

Lemma 4.2. Let $x, y$ be Boolean independent selfadjoint random variables from $(\mathcal{A}, \mathbb{E})$. Let $b \in \mathcal{B}$ be such that $x+y-b$ and $y-b$ are invertible. Then

$$
\mathbb{E}\left[(b-x-y)^{-1}\right]=\left(1+\mathbb{E}\left[(b-x-y)^{-1} x\right]\right) \mathbb{E}\left[(y-b)^{-1}\right]
$$

Proof. Notice that $(b-x-y)^{-1}$ is contained in the norm closure of $\operatorname{alg}\{\mathcal{B}, x, y\}$ and $(b-y)^{-1}$ in the norm closure of $\operatorname{alg}\{\mathcal{B}, y\}$. By the the norm continuity of $\mathbb{E}$ and Lemma 4.1, we have

$$
\mathbb{E}\left[(b-x-y)^{-1} x(b-y)^{-1}\right]=\mathbb{E}\left[(b-x-y)^{-1} x\right] \mathbb{E}\left[(b-y)^{-1}\right] .
$$

On the other hand, we have

$$
(b-x-y)^{-1}=(b-y)^{-1}+(b-x-y)^{-1} x(b-y)^{-1} .
$$

It follows that

$$
\begin{aligned}
\mathbb{E}\left[(b-x-y)^{-1}\right] & =\mathbb{E}\left[(b-y)^{-1}+(b-x-y)^{-1} x(b-y)^{-1}\right] \\
& =\left(1+\mathbb{E}\left[(b-x-y)^{-1} x\right]\right) \mathbb{E}\left[(b-y)^{-1}\right]
\end{aligned}
$$

The proof is done.

Lemma 4.3. Let $\mu, \nu \in \Sigma_{0}(\mathcal{B})$. Then

$$
F_{\mu \uplus \nu, 1}(b)=F_{\mu, 1}(b)+F_{\nu, 1}(b)-b,
$$

for $b \in \mathbb{H}^{+}$.

Proof. Since $\mu, \nu \in \Sigma_{0}(\mathcal{B})$, there exist Boolean independent selfadjoint random variables $x, y$ from a $\mathcal{B}$-valued probability space $(\mathcal{A}, \mathbb{E})$ such that $\mu$ equals the distribution of $x$ and $\nu$ equals the distribution of $y$. By Lemma 4.2, we have

$$
\begin{aligned}
G_{\mu \uplus \nu, 1}(b) F_{\nu, 1}(b) & =\mathbb{E}\left[(b-x-y)^{-1}\right] \mathbb{E}\left[(b-y)^{-1}\right]^{-1} \\
& =\left(1+\mathbb{E}\left[(b-x-y)^{-1} x\right]\right) \mathbb{E}\left[(b-y)^{-1}\right] \mathbb{E}\left[(b-y)^{-1}\right]^{-1} \\
& =1+\mathbb{E}\left[(b-x-y)^{-1} x\right] .
\end{aligned}
$$


Similarly, we have $G_{\mu \uplus \nu, 1}(b) F_{\mu, 1}(b)=1-\mathbb{E}\left[(b-x-y)^{-1} y\right]$. Therefore, we have

$$
\begin{aligned}
& G_{\mu \uplus \nu, 1}(b)\left(F_{\mu}(b)+F_{\nu, 1}(b)-b\right) \\
= & 2+\mathbb{E}\left[(b-x-y)^{-1} x\right]+\mathbb{E}\left[(b-x-y)^{-1} y\right]-\mathbb{E}\left[(b-x-y)^{-1} b\right] \\
= & 1 .
\end{aligned}
$$

Since $F_{\mu \uplus \nu, 1}(b)=G_{\mu \uplus \nu, 1}(b)^{-1}$, the statement follows.

Notice that the Boolean additive convolution is a kind of $\mathcal{J}$ additive convolution, the $\mathcal{B} \otimes M_{n}(\mathbb{C})$-valued distribution of $x \otimes I_{n}+y \otimes I_{n}$ is then the Boolean additive convolution of the distributions of $x \otimes I_{n}$ and $y \otimes I_{n}$. Therefore, we have the following result.

Proposition 4.4. Let $\mu, \nu \in \Sigma_{0}(\mathcal{B})$. Then, for all $n \in \mathbb{N}$, we have

$$
F_{\mu \uplus \nu, n}\left(b_{n}\right)=F_{\mu, n}\left(b_{n}\right)+F_{\nu, n}\left(b_{n}\right)-b_{n},
$$

for $b_{n} \in \mathbb{H}_{n}^{+}$.

4.2. Operator valued Boolean convolution Powers. In this subsection, we study the reciprocal Cauchy transforms for $\mathcal{B}$-valued Boolean convolution Powers.

Definition 4.5. Let $S$ be an ordered set. A partition $\sigma$ of a set $S$ is a collection of disjoint nonempty sets $V_{1}, \ldots, V_{r}$ whose union is $S$. $V_{1}, \ldots, V_{r}$ are called blocks of $\sigma$. A block $V$ of $\sigma$ is an interval if there is no triple $\left(s_{1}, s_{2}, r\right)$ such that $s_{1}<r<s_{2}, s_{1}, s_{2} \in V, r \notin V$. A partition $\sigma \in P(S)$ is an interval partition if every block of $\sigma$ is an interval. The family of interval partitions of $S$ will be denoted by $I N(S)$. Let $l<n$ be two natural numbers, we denote by $[l, n]$ the interval $\{l, l+1, \cdots, k\}$ and denote by $[n]$ the interval $[1, n]$.

Definition 4.6. A $\mathcal{B}$-series $\left(\beta^{[n]}\right)_{n \geqslant 1}$ is a sequence of $\mathbb{C}$-multilinear maps that, for each $n \geqslant 1, \beta^{[n]}: \mathcal{B}^{n-1} \rightarrow \mathcal{B}$. Given an interval partition $\sigma \in I N(n)$, then $\beta^{[\sigma]}$ is a $\mathbb{C}$-multilinear map form $\mathcal{B}^{n-1}$ to $\mathcal{B}$ defined recursively as follows: If $\sigma$ contains only one block $[n]$, then $\beta^{[\sigma]}=\beta^{[n]}$. Otherwise, suppose that $[k+1, n]$ is an interval block of $\sigma$ and let $\sigma^{\prime}$ be the restriction of $\sigma$ to the interval $[1, k]$. Then

$$
\beta^{[\sigma]}\left(b_{1}, \cdots, b_{n-1}\right)=\beta^{\left[\sigma^{\prime}\right]}\left(b_{1}, \cdots, b_{k-1}\right) b_{k} \beta^{[n-k]}\left(b_{k+1}, \cdots, b_{n-1}\right) .
$$

Remark 4.7. Notice that $\beta_{1}$ is always a $\mathcal{B}$-constant. The partitions in the previous definition can be replaced by noncrossing partitions which are very useful combinatorial tools in studying free independence relation.

Definition 4.8. Given a random variable $x \in(\mathcal{A}, \mathbb{E})$, the moment series of $x$ is a $\mathcal{B}$-series $\left(M_{x}^{[n]}\right)_{n \geqslant 1}$ that $M_{x}^{[1]}=\mathbb{E}[x]$ and

$$
M_{x}^{[n]}\left(b_{1}, \cdots, b_{n-1}\right)=\mathbb{E}\left[x b_{1} x \cdots x b_{n-1} x\right],
$$


for $n \geqslant 2$ and $b_{1}, \cdots, b_{n}-1 \in \mathcal{B}$. The B-transform of $\left(M_{x}^{[n]}\right)_{n \geqslant 1}$ is the series $\left(B_{x}^{[n]}\right)_{n \geqslant 1}$, such that

$$
M_{x}^{[n]}\left(b_{1}, \cdots, b_{n-1}\right)=\sum_{\sigma \in I N(n)} B_{x}^{[\sigma]}\left(b_{1}, \cdots, b_{n-1}\right)
$$

Remark 4.9. $\left(B_{x}^{[n]}\right)_{n \geqslant 1}$ can be solved recursively as follows: $B_{x}^{[1]}=\mathbb{E}[x]$ and

$$
B_{x}^{[n]}\left(b_{1}, \cdots, b_{n-1}\right)=\mathbb{E}\left[x b_{1} x \cdots x b_{n-1} x\right]-\sum_{\sigma \in I N(n), \sigma \neq\{[n]\}} B_{x}^{[\sigma]}\left(b_{1}, \cdots, b_{n-1}\right),
$$

for $n \geqslant 2$.

Definition 4.10. Given $\mu \in \Sigma_{0}(\mathcal{B})$ such that $\mu$ is equal to the distribution of $x$ in $(\mathcal{A}, \mathbb{E})$, then we set

$$
B_{\mu}^{[\sigma]}=B_{x}^{[\sigma]}
$$

for all $\sigma \in I N(n), n \in \mathbb{N}$. Let $\alpha$ be a completely positive map. Then $\mu^{\uplus \alpha}$ is a distribution such that

$$
B_{\mu^{\uplus \alpha}}^{[n]}=\alpha\left(B_{\mu}^{[n]}\right)
$$

for all $n \in \mathbb{N}$.

The following definition is from Popa [24], and is developed more in [25].

Definition 4.11. Let $x \in(\mathcal{A}, \mathbb{E})$ be a selfadjoint random variable. The Boolean cumulants of $x$ are a family of $\mathcal{B}$-multilinear maps $\left\{B_{n, x}\right\}_{n \geqslant 1}$, such that $B_{n, x}: \mathcal{B}^{n} \rightarrow \mathcal{B}$ and

$$
\mathbb{E}\left[x b_{1} \cdots x b_{n}\right]=\sum_{k=0}^{n-1} \mathbb{E}\left[x b_{1} \cdots x b_{k}\right] B_{n-k, x}\left(b_{k+1}, \cdots, b_{n}\right) .
$$

Lemma 4.12. Given $x \in(\mathcal{A}, \mathbb{E})$, we have

$$
B_{n, x}\left(b_{1}, \cdots, b_{n}\right)=B_{x}^{[n]}\left(b_{1}, \cdots, b_{n-1}\right) b_{n} .
$$


Proof. When $n=1$, we have $B_{1, x}\left(b_{1}\right)=\mathbb{E}\left[x b_{1}\right]=\mathbb{E}[x] b_{1}=B_{x}^{[1]} b_{1}$.

When $n \geqslant 2$, we have

$$
\begin{aligned}
& B_{n, x}\left(b_{1}, \cdots, b_{n}\right) \\
= & \mathbb{E}\left[x b_{1} x \cdots b_{n-1} x b_{n}\right]-\sum_{k=1}^{n-1} \mathbb{E}\left[x b_{1} \cdots x b_{k}\right] B_{n-k, x}\left(b_{k+1}, \cdots, b_{n}\right) \\
= & \mathbb{E}\left[x b_{1} x \cdots b_{n-1} x\right] b_{n}-\sum_{k=1}^{n-1} \mathbb{E}\left[x b_{1} \cdots b_{k-1} x\right] b_{k} B_{x}^{n-k}\left(b_{k+1}, \cdots, b_{n-1}\right) b_{n} \\
= & \left(\mathbb{E}\left[x b_{1} x \cdots b_{n-1} x\right]-\sum_{k=1}^{n-1} \mathbb{E}\left[x b_{1} \cdots b_{k-1} x\right] b_{k} B_{x}^{[n-k]}\left(b_{k+1}, \cdots, b_{n-1}\right)\right) b_{n} \\
= & \left(\mathbb{E}\left[x b_{1} x \cdots b_{n-1} x\right]-\sum_{k=1}^{n-1} \sum_{\sigma^{\prime} \in I N(k)} B_{x}^{\left[\sigma^{\prime}\right]}\left(b_{1}, \cdots, b_{k-1}\right) b_{k} B_{x}^{[n-k]}\left(b_{k+1}, \cdots, b_{n-1}\right)\right) b_{n} \\
= & \left.\left(\mathbb{E}\left[x b_{1} x \cdots b_{n-1} x\right]-\sum_{\sigma \in I N(n), \sigma \neq\{[n]\}} B_{x}^{[\sigma]}\left(b_{1}, \cdots, b_{n-1}\right)\right)\right) b_{n} \\
= & B_{x}^{[n]}\left(b_{1}, \cdots, b_{n-1}\right) b_{n} .
\end{aligned}
$$

The proof is done.

Let $(\mathcal{M}, \xi)$ be a $\mathcal{B}-\mathcal{B}$ bimodule with a specified vector $\xi, x$ be a random variable in $\left(L(\mathcal{M}), \phi_{\xi}\right)$. Let $\mathcal{M}=\mathcal{B} \xi \oplus \mathcal{M}^{\circ}$ where $\mathcal{M}^{\circ}=(\mathcal{B} \xi)^{\perp}$. We write $x$ in the following matrix form

$$
x=\left(\begin{array}{cc}
p & a \\
a^{*} & T
\end{array}\right),
$$

where $a: \mathcal{B} \xi \rightarrow \mathcal{M}^{\circ}, T: \mathcal{M}^{\circ} \rightarrow \mathcal{M}^{\circ}, p: \mathcal{B} \xi \rightarrow \mathcal{B} \xi$.

By Lemma 3.9 of [25] and Lemma 4.12, we have $B_{x}^{[1]}=\mathbb{E}[x]$ and

$$
B_{x}^{[n]}\left(b_{1}, \cdots, b_{n-1}\right)=B_{n, x}\left(b_{1}, \cdots b_{n-1}, 1_{\mathcal{B}}\right)=\left\langle a^{*} b_{1} T b_{2} \cdots T b_{n-1} a \xi, \xi\right\rangle,
$$

for $n \geqslant 2$.

Let $\mathcal{H}$ be a $\mathcal{B}-\mathcal{B}$ Hilbert bimodule and $\zeta \in \mathcal{H}$ be such that for $b \in \mathcal{B}$, we have

$$
\langle\zeta, b \zeta\rangle_{\mathcal{H}}=\alpha(b),
$$

where $\langle\cdot, \cdot\rangle_{\mathcal{H}}$ is the inner product on $\mathcal{H}$.

Let $\mathcal{K}=\mathcal{B} \xi \oplus \mathcal{M}^{\circ} \otimes_{\mathcal{B}} \mathcal{H}$ with the inner product

$$
\left\langle b \xi \oplus m \otimes_{\mathcal{B}} h, b^{\prime} \xi \oplus m^{\prime} \otimes_{\mathcal{B}} h^{\prime}\right\rangle_{\mathcal{K}}=b^{*} b^{\prime}+\left\langle h,\left\langle m, m^{\prime}\right\rangle_{\mathcal{M}} h^{\prime}\right\rangle_{\mathcal{H}},
$$

for $b, b^{\prime} \in \mathcal{B}, m, m^{\prime} \in \mathcal{M}^{\circ}$ and $h, h^{\prime} \in \mathcal{H}$. 
Notice that $b \xi=\xi b$, the following maps are well defined. Let $\tilde{a}, \tilde{p}, \tilde{T}: \mathcal{K} \rightarrow \mathcal{K}$ be such that

$$
\begin{gathered}
\tilde{a}\left(b \xi \oplus m \otimes_{\mathcal{B}} h\right)=a(\xi) \otimes_{\mathcal{B}}(\zeta b), \\
\tilde{p}\left(b \xi \oplus m \otimes_{\mathcal{B}} h\right)=\alpha(p) b \xi, \\
\tilde{T}^{*}\left(b \xi \oplus m \otimes_{\mathcal{B}} h\right)=T m \otimes_{\mathcal{B}} h,
\end{gathered}
$$

for all $b \in \mathcal{B}, m \in \mathcal{M}^{\circ}$ and $h \in \mathcal{H}$.

Lemma 4.13. $\tilde{a} \in L(\mathcal{K})$ and

$$
\tilde{a}^{*}\left(b \xi \oplus m \otimes_{\mathcal{B}} h\right)=\left\langle\zeta, a^{*}(m) h\right\rangle_{\mathcal{H}} \xi .
$$

Proof. Notice that $\langle a(b \xi), m\rangle_{\mathcal{M}}=b^{*} a^{*}(m)$, we have

$$
\begin{aligned}
& \left\langle\tilde{a}\left(b \xi \oplus m \otimes_{\mathcal{B}} h\right), b^{\prime} \xi \oplus m^{\prime} \otimes_{\mathcal{B}} h^{\prime}\right\rangle_{\mathcal{K}} \\
= & \left\langle a(\xi) \otimes_{\mathcal{B}}(\zeta b), b^{\prime} \xi \oplus m^{\prime} \otimes_{\mathcal{B}} h^{\prime}\right\rangle_{\mathcal{K}} \\
= & \left\langle\zeta b,\left\langle a(b \xi), m^{\prime}\right\rangle_{M} h^{\prime}\right\rangle_{\mathcal{H}} \\
= & b^{*}\left\langle\zeta,\left\langle a(\xi), m^{\prime}\right\rangle_{M} h^{\prime}\right\rangle_{h} h \\
= & b^{*}\left\langle\zeta, a^{*}\left(m^{\prime}\right) h^{\prime}\right\rangle_{h} h \\
= & b^{*} \tilde{a}^{*}\left(b^{\prime} \xi \oplus m^{\prime} \otimes_{\mathcal{B}} h^{\prime}\right) \\
= & \left\langle b \xi \oplus m \otimes_{\mathcal{B}} h, \tilde{a}^{*}\left(b^{\prime} \xi \oplus m^{\prime} \otimes_{\mathcal{B}} h^{\prime}\right)\right\rangle_{\mathcal{K}} .
\end{aligned}
$$

The proof is done.

Lemma 4.14. Let $\tilde{x}=\tilde{a}+\tilde{a}^{*}+\tilde{p}+\tilde{T}$. Then $\tilde{x} \in L(\mathcal{K})$, namely $\tilde{x}$ is a selfadjointable operator. Moreover, $\tilde{x}=\tilde{x}^{*}$.

Proof. It suffice to show that

$$
\left\langle\tilde{x}\left(b \xi \oplus m \otimes_{\mathcal{B}} h\right), b^{\prime} \xi \oplus m^{\prime} \otimes_{\mathcal{B}} h^{\prime}\right\rangle_{\mathcal{K}}=\left\langle b \xi \oplus m \otimes_{\mathcal{B}} h, \tilde{x}\left(b^{\prime} \xi \oplus m^{\prime} \otimes_{\mathcal{B}} h^{\prime}\right)\right\rangle_{\mathcal{K}},
$$

for $b, b^{\prime} \in \mathcal{B}, m, m^{\prime} \in \mathcal{M}^{\circ}$ and $h, h^{\prime} \in H$.

Since $x$ is selfadjoint, we have $T$ is selfadjoint in $L(M)$ and

$$
\begin{aligned}
\left\langle\tilde{T}\left(b \xi \oplus m \otimes_{\mathcal{B}} h\right), b^{\prime} \xi \oplus m^{\prime} \otimes_{\mathcal{B}} h^{\prime}\right\rangle_{\mathcal{K}} & =\left\langle h,\left\langle m, T^{*} m^{\prime}\right\rangle_{\mathcal{M}} h^{\prime}\right\rangle_{\mathcal{H}} \\
& =\left\langle h,\left\langle m, T m^{\prime}\right\rangle_{\mathcal{M}} h^{\prime}\right\rangle_{\mathcal{H}} \\
& =\left\langle b \xi \oplus m \otimes_{\mathcal{B}} h, \tilde{T}\left(b^{\prime} \xi \oplus m^{\prime} \otimes_{\mathcal{B}} h^{\prime}\right)\right\rangle_{\mathcal{K}} .
\end{aligned}
$$

Since $p \in \mathcal{B}$ is selfadjoint, we have

$$
\begin{aligned}
\left\langle\tilde{p}\left(b \xi \oplus m \otimes_{\mathcal{B}} h\right), b^{\prime} \xi \oplus m^{\prime} \otimes_{\mathcal{B}} h^{\prime}\right\rangle_{\mathcal{K}} & =b^{*} \alpha(p)^{*} b^{\prime} \\
& =b^{*} \alpha(p) b^{\prime} \\
& =\left\langle b \xi \oplus m \otimes_{\mathcal{B}} h, \tilde{p}\left(b^{\prime} \xi \oplus m^{\prime} \otimes_{\mathcal{B}} h^{\prime}\right)\right\rangle_{\mathcal{K}}
\end{aligned}
$$


Together with Lemma 4.13, the proof is done.

Proposition 4.15. Let $\tilde{\mu}$ be the distribution of $\tilde{x}$ with respect to $\langle\xi, \xi\rangle_{\mathcal{K}}$. Then,

$$
\tilde{\mu}=\mu^{\uplus \alpha}
$$

where $\mu$ is the distribution of $x$ with respect to $\langle\xi, \xi\rangle_{\mathcal{M}}$

Proof. By Lemma 3.9, we that $B_{x}^{[1]}=B_{1, x}\left(1_{\mathcal{B}}\right)=p$ and $B_{\tilde{x}}^{[1]}=B_{1, \tilde{x}}\left(1_{\mathcal{B}}\right)=\alpha(p)=\alpha\left(B_{x}^{[1]}\right)$.

For $n \geqslant 2$, we have

$$
\begin{aligned}
B_{x}^{[n]}\left(b_{1}, \cdots, b_{n-1}\right) & =B_{n, x}\left(b_{1}, \cdots, b_{n-1}, 1_{\mathcal{B}}\right) \\
& =\left\langle\xi, a^{*} b_{1} T b_{2} T \cdots b_{n-1} T a \xi\right\rangle \\
& =a^{*} b_{1} T b_{2} T \cdots b_{n-1} T a
\end{aligned}
$$

and

$$
\begin{aligned}
B_{\tilde{x}}^{[n]}\left(b_{1}, \cdots, b_{n-1}\right) & =B_{n, \tilde{x}}\left(b_{1}, \cdots, b_{n-1}, 1_{\mathcal{B}}\right) \\
& =\left\langle\xi, \tilde{a}^{*} b_{1} \tilde{T} b_{2} \tilde{T} \cdots b_{n-1} \tilde{T} \tilde{a} \xi\right\rangle_{\mathcal{K}} \\
& =\left\langle\xi, \tilde{a}^{*} b_{1} \tilde{T} b_{2} \tilde{T} \cdots b_{n-1} \tilde{T} \tilde{a} \xi\right\rangle_{\mathcal{K}} \\
& =\left\langle\xi \tilde{a}^{*}\left(b_{1} \tilde{T} b_{2} \tilde{T} \cdots b_{n-1} \tilde{T} a(\xi) \otimes \zeta\right)\right\rangle_{\mathcal{K}} \\
& =\left\langle\xi \tilde{a}^{*}\left[\left(b_{1} T b_{2} T \cdots b_{n-1} T a(\xi)\right) \otimes \zeta\right]\right\rangle_{\mathcal{K}} \\
& =\left\langle\xi,\left\langle\zeta, a^{*}\left(b_{1} T b_{2} T \cdots b_{n-1} T a(\xi)\right) \zeta\right\rangle_{\mathcal{H}}\right\rangle_{\mathcal{K}} \\
& =\left\langle\xi, \alpha\left(a^{*} b_{1} T b_{2} T \cdots b_{n-1} T a\right) \xi\right\rangle_{\mathcal{K}} \\
& =\alpha\left(a^{*} b_{1} T b_{2} T \cdots b_{n-1} T a\right) \\
& =\alpha\left(B_{x}^{[n]}\left(b_{1}, \cdots, b_{n-1}\right)\right) .
\end{aligned}
$$

The proof is done. 
For $b \in \mathbb{H}^{+}$, by Proposition 2.14, we have that $F_{\mu_{x}, 1}(b)=b-p-a^{*}(b-T)^{-1} a$, thus

$$
\begin{aligned}
F_{\tilde{\mu}, 1}(b) & =\tilde{p}-b-\tilde{a}^{*}(\tilde{T}-b)^{-1} \tilde{a} \\
& =b-\tilde{p}-\left\langle\xi, \tilde{a}^{*}(b-\tilde{T})^{-1} \tilde{a} \xi\right\rangle_{\mathcal{K}} \\
& =b-\tilde{p}-\left\langle a(\xi) \otimes \zeta,(b-\tilde{T})^{-1}(a(\xi) \otimes \zeta)\right\rangle_{\mathcal{K}} \\
& =b-\tilde{p}-\left\langle a(\xi) \otimes \zeta,\left((b-T)^{-1} a(\xi)\right) \otimes \zeta\right\rangle_{\mathcal{K}} \\
& =b-\tilde{p}-\left\langle\zeta,\left\langle a(\xi),\left((b-T)^{-1} a(\xi)\right)\right\rangle_{\mathcal{M}} \zeta\right\rangle_{\mathcal{H}} \\
& =b-\alpha(p)-\alpha\left(\left\langle a(\xi),\left((b-T)^{-1} a(\xi)\right)\right\rangle_{\mathcal{M}}\right) \\
& =b-\alpha(p)-\alpha\left(a^{*}(T-b)^{-1} a\right) \\
& =\alpha\left(F_{\mu, 1}(b)\right)+b-\alpha(b) .
\end{aligned}
$$

Therefore, we have the following result.

Lemma 4.16. Let $\mu$ be a $\mathcal{B}$-valued distribution and let $\alpha$ be a completely positive map from $\mathcal{B}$ to $\mathcal{B}$. Then,

$$
F_{\mu^{\uplus \alpha}, 1}(b)=\alpha\left(F_{\mu, 1}(b)\right)+(1-\alpha)(b),
$$

for all $b \in \mathbb{H}^{+}$.

Similarly following the previous construction, we have the following result.

Theorem 4.17. Let $\mu \in \Sigma_{0}(\mathcal{B})$ be a $\mathcal{B}$-valued distribution and let $\alpha$ be a completely positive map from $\mathcal{B}$ to $\mathcal{B}$. For each $n \in \mathbb{N}$, we have

$$
F_{\mu^{\uplus \alpha, n}}\left(b_{n}\right)=\alpha_{n}\left(F_{\mu, n}\left(b_{n}\right)\right)+\left(1_{n}-\alpha_{n}\right)\left(b_{n}\right),
$$

for all $b \in \mathbb{H}_{n}^{+}$and $\alpha_{n}=\alpha \otimes I_{n}$ is a map from $\mathcal{B} \otimes M_{n}(\mathbb{C})$ to $\mathcal{B} \otimes M_{n}(\mathbb{C})$ such that $\alpha_{n}(b \otimes e)=\alpha(b) \otimes e$ for all $b \in \mathcal{B}$ and $e \in M_{n}(\mathbb{C})$.

\section{Monotone Convolutions}

In this section, we study some analytic properties of the $\mathcal{B}$-valued monotone additve convolution. The combinatorial aspects of $\mathcal{B}$-valued monotone additive convolution are studied in [23]. Given $x \in(\mathcal{A}, \mathbb{E})$, we denote by $\operatorname{alg}_{0}\{x, \mathcal{B}\}=\left\{p(x) \mid p \in \mathcal{B}\langle X\rangle_{0}\right\}$ and $\operatorname{alg}\{x, \mathcal{B}\}=\{p(x) \mid p \in \mathcal{B}\langle X\rangle\}$.

Lemma 5.1. Let $x, y \in(\mathcal{A}, \mathbb{E})$ be two $\mathcal{B}$-valued random variables such that $x, y$ are monotone. Then $\operatorname{alg}_{0}\{x, \mathcal{B}\}$ and $\operatorname{alg}\{y, \mathcal{B}\}$ are monotone independent.

Proof. Let $a_{1}, \cdots, a_{n} \in \operatorname{alg}\{x, \mathcal{B}\} \cup \operatorname{alg}\{y, \mathcal{B}\}$ such that $a_{k} \in \operatorname{alg}\{y, \mathcal{B}\}$ and $a_{k-1}, a_{k+1} \in$ $\operatorname{alg}_{0}\{x, \mathcal{B}\}$. Then $a_{k}=a_{k}^{\prime}+b$ for some $a_{k}^{\prime} \in a l g_{0}\{y, \mathcal{B}\}$ and $b \in \mathcal{B}$. Since $x, y$ are monotone independent, we have

$$
\mathbb{E}\left[a_{1} \cdots a_{k-1} a_{k}^{\prime} a_{k+1} a_{n}\right]=\mathbb{E}\left[\left[a_{1} \cdots a_{k-1} \mathbb{E}\left[a_{k}^{\prime}\right] a_{k+1} a_{n}\right]\right.
$$


On the other hand,

$$
\mathbb{E}\left[a_{1} \cdots a_{k-1} b a_{k+1} a_{n}\right]=\mathbb{E}\left[\left[a_{1} \cdots a_{k-1} \mathbb{E}[b] a_{k+1} a_{n}\right]\right.
$$

Therefore,

$$
\mathbb{E}\left[a_{1} \cdots a_{k-1} a_{k} a_{k+1} a_{n}\right]=\mathbb{E}\left[\left[a_{1} \cdots a_{k-1} \mathbb{E}\left[a_{k}\right] a_{k+1} a_{n}\right] .\right.
$$

Since $a_{1}, \cdots, a_{n} \in \operatorname{alg}_{0}\{x, \mathcal{B}\} \cup \operatorname{alg}\{y, \mathcal{B}\}$ are arbitrary, the proof is done.

Lemma 5.2. Let $x, y \in(\mathcal{A}, \mathbb{E})$ be two $\mathcal{B}$-valued random variables such that $x, y$ are monotone. Given $a \in \operatorname{alg}(x, y, \mathcal{B}), x_{1} \in \operatorname{alg} g_{0}\{x, \mathcal{B}\}, x_{2} \in \operatorname{alg}\{x, \mathcal{B}\}$ and $y^{\prime} \in \operatorname{alg}\{y, \mathcal{B}\}$, then

$$
\mathbb{E}\left[a x_{1} y^{\prime} x_{2}\right]=\mathbb{E}\left[a x_{1} \mathbb{E}\left[y^{\prime}\right] x_{2}\right]
$$

Proof. Since $x_{2} \in \operatorname{alg}\{x, \mathcal{B}\}$, there exists $x_{2}^{\prime} \in \operatorname{alg}\{x, \mathcal{B}\}$ such that $x_{2}=x_{2}^{\prime}+b$ for some $b \in \mathcal{B}$. Therefore,

$$
\begin{aligned}
\mathbb{E}\left[a x_{1} y^{\prime} x_{2}\right] & =\mathbb{E}\left[a x_{1} y^{\prime}\left(x_{2}^{\prime}+b\right)\right] \\
& =\mathbb{E}\left[a x_{1} y^{\prime} x_{2}^{\prime}\right]+\mathbb{E}\left[a x_{1} y b\right] \\
& =\mathbb{E}\left[a x_{1} \mathbb{E}\left[y^{\prime}\right] x_{2}^{\prime}\right]+\mathbb{E}\left[a x_{1}\right] \mathbb{E}\left[y^{\prime} b\right] \\
& =\mathbb{E}\left[a x_{1} \mathbb{E}\left[y^{\prime}\right] x_{2}^{\prime}\right]+\mathbb{E}\left[a x_{1} \mathbb{E}\left[y^{\prime}\right] b\right] \\
& =\mathbb{E}\left[a x_{1} \mathbb{E}\left[y^{\prime}\right] x_{2}\right],
\end{aligned}
$$

which is desired.

Lemma 5.3. Let $x, y \in(\mathcal{A}, \mathbb{E})$ be two $\mathcal{B}$-valued selfadjoint random variables such that $x, y$ are monotone. For $b \in \mathbb{H}^{+}$, we have

$$
\left.\mathbb{E}\left[(b-y)(b-x-y)^{-1}\right]\right)=\mathbb{E}\left[\left(1-x G_{y}(b)\right)^{-1}\right]
$$

Proof. Since $x, y$ are selfadjoint and $b \in \mathbb{H}^{+}, b-y$ and $b-x-y$ are invertible. It follows that

$$
\left.\left.\mathbb{E}\left[(b-y)(b-x-y)^{-1}\right]\right)=\mathbb{E}\left[\left(1-x(b-y)^{-1}\right)^{-1}\right]\right) .
$$

Therefore, we have

$$
\begin{aligned}
& \left.\mathbb{E}\left[\left(1-x(b-y)^{-1}\right)^{-1}\right]\right)-\mathbb{E}\left[\left(1-x G_{y}(b)\right)^{-1}\right] \\
= & \mathbb{E}\left[\left(1-x(b-y)^{-1}\right)^{-1}\left(x G_{y}(b)-x(b-y)^{-1}\right)\left(1-x G_{y}(b)\right)^{-1}\right] \\
= & \mathbb{E}\left[\left(1-x(b-y)^{-1}\right)^{-1}\left(x\left(G_{y}(b)-(b-y)^{-1}\right)\right)\left(1-x G_{y}(b)\right)^{-1}\right] .
\end{aligned}
$$


Notice that $\left(1-x G_{y}(b)\right)^{-1} \in \overline{\operatorname{alg}\{x, \mathcal{B}\}}^{\|\cdot\|},\left(G_{y}(b)-(b-y)^{-1}\right) \in \overline{\operatorname{alg}\{y, \mathcal{B}\}}^{\|\cdot\|}, \mathbb{E}\left[\left(G_{y}(b)-\right.\right.$ $\left.\left.(b-y)^{-1}\right)\right]=0$ and $\mathbb{E}$ is norm-continuous, we have that

$$
\begin{aligned}
& \mathbb{E}\left[\left(1-x(b-y)^{-1}\right)^{-1} x\left(G_{y}(b)-(b-y)^{-1}\right)\left(1-x G_{y}(b)\right)^{-1}\right] \\
= & \mathbb{E}\left[\left(1-x(b-y)^{-1}\right)^{-1} x \mathbb{E}\left[G_{y}(b)-(b-y)^{-1}\right]\left(1-x G_{y}(b)\right)^{-1}\right] \\
= & 0 .
\end{aligned}
$$

The proof is done.

Lemma 5.4. Let $\mu, \nu \in \Sigma_{0}(\mathcal{B})$. Then,

$$
F_{\mu \triangleright \nu, 1}(b)=F_{\mu, 1}\left(F_{\nu, 1}(b)\right)
$$

for $b \in \mathbb{H}^{+}$.

Proof. Let $x, y$ be monotone independent selfadjoint random variables from a $\mathcal{B}$-valued probability space $(\mathcal{A}, \mathbb{E})$ such that $\mu$ equals the distribution of $x$ and $\nu$ equals the distribution of $y$. By Lemma 5.3, we have

$$
\begin{aligned}
G_{\mu \triangleright \nu, 1}(b) & =\mathbb{E}\left[(b-x-y)^{-1}\right] \\
& =\mathbb{E}\left[(b-y)^{-1}\right]+\mathbb{E}\left[(b-y)^{-1} x(b-x-y)^{-1}\right] \\
& =\mathbb{E}\left[(b-y)^{-1}\right]+\mathbb{E}\left[(b-y)^{-1}\right] \mathbb{E}\left[x(b-x-y)^{-1}\right] \\
& =\mathbb{E}\left[(b-y)^{-1}\right]\left[1_{\mathcal{B}}+\mathbb{E}\left[x(b-x-y)^{-1}\right]\right. \\
& =\mathbb{E}\left[(b-y)^{-1}\right]\left(1_{\mathcal{B}}+\mathbb{E}\left[x(b-x-y)^{-1}\right]\right) \\
& =\mathbb{E}\left[(b-y)^{-1}\right]\left(\mathbb{E}\left[(b-y)(b-x-y)^{-1}\right]\right) \\
& =G_{\nu, 1}(b) \mathbb{E}\left[\left(1-x G_{\nu, 1}(b)\right)^{-1}\right] \\
& =\mathbb{E}\left[\left(G_{\nu, 1}(b)^{-1}-x\right)^{-1}\right] \\
& =G_{\mu, 1}\left(F_{\nu, 1}(b)\right) .
\end{aligned}
$$

The proof is done.

Notice that monotone additive convolution is a kind of $\mathcal{J}$-additive convolution, the $\mathcal{B} \otimes M_{n}(\mathbb{C})$-valued distribution of $x \otimes I_{n}+y \otimes I_{n}$ is then the monotone additive convolution of the distributions of $x \otimes I_{n}$ and $y \otimes I_{n}$. Therefore, we have the following result.

Proposition 5.5. Let $\mu, \nu \in \Sigma_{0}(\mathcal{B})$. For $n \in \mathbb{N}$, we have

$$
F_{\mu \triangleright \nu, n}\left(b_{n}\right)=F_{\mu, n}\left(F_{\nu, n}\left(b_{n}\right)\right),
$$

for $b_{n} \in \mathbb{H}_{n}^{+}$. 


\section{Orthogonal Convolution}

In this section, we study some analytic properties of the orthogonal additive convolutions. Let $\mu_{1}, \mu_{2} \in \Sigma_{0}(\mathcal{B})$. For $i=1,2$, let $\left(\mathcal{M}_{i}, \xi_{i}\right)$ be a $\mathcal{B}$ - $\mathcal{B}$ bimodules with specified vectors and $x_{i} \in\left(L\left(\mathcal{M}_{i}\right), \phi_{\xi_{i}}\right)$ such that $\mu_{i}$ is equal to the distribution of $x_{i}$. Let $(\mathcal{M}, \xi)$ be the reduced free product of $\left(\mathcal{M}_{i}, \xi\right)$ and $\lambda_{i}: L\left(\mathcal{M}_{i}\right) \rightarrow L(M)$ are the embedding isomorphisms defined in Section 3. Let $\mathcal{J}_{1}=\{\varnothing,(1)\}$ and $\mathcal{J}_{2}=\{(1),(2,1)\}$. Then, $P_{\mathcal{J}_{1}}^{\mathcal{M}}$ is the orthogonal projection onto

$$
\mathcal{B}_{\xi} \oplus \mathcal{M}_{1}^{\circ}
$$

and $P_{\mathcal{J}_{1}}^{\mathcal{M}}$ is the orthogonal projection onto

$$
\mathcal{M}_{1}^{\circ} \oplus \mathcal{M}_{2}^{\circ} \otimes_{\mathcal{B}} \mathcal{M}_{1}^{\circ}
$$

Then, the orthogonal additive convolution $\mu_{1} \vdash \mu_{2}$ of $\mu$ and $\nu$ is the distribution of $P_{\mathcal{J}_{1}}^{\mathcal{M}} \lambda_{1}\left(x_{1}\right)+P_{\mathcal{J}_{2}}^{\mathcal{M}} \lambda_{2}\left(x_{2}\right)$ in $\left(L(\mathcal{M}), \phi_{\xi}\right)$.

Notice that $P_{\mathcal{J}_{1}}^{\mathcal{M}}$ and $P_{\mathcal{J}_{2}}^{\mathcal{M}}$ are projections from $\mathcal{M}$ into

$$
\mathcal{M}_{\vdash}=\mathcal{B} \xi \oplus \mathcal{M}_{1}^{\circ} \oplus \mathcal{M}_{2}^{\circ} \otimes_{\mathcal{B}} \mathcal{M}_{1}^{\circ}
$$

and $\left(\mathcal{M}_{\vdash}, \xi\right)$ is a $\mathcal{B}$ - $\mathcal{B}$ bimodules with specified vector, it follows that the distribution of $P_{\mathcal{J}_{1}}^{\mathcal{M}} \lambda_{1}\left(x_{1}\right)+P_{\mathcal{J}_{2}}^{\mathcal{M}} \lambda_{2}\left(x_{2}\right)$ in $\left(L(\mathcal{M}), \phi_{\xi}\right)$ is equal to the distribution of $P_{\mathcal{J}_{1}}^{\mathcal{M}} \lambda_{1}\left(x_{1}\right)+P_{\mathcal{J}_{2}}^{\mathcal{M}} \lambda_{2}\left(x_{2}\right)$ in $\left(L\left(\mathcal{M}_{\vdash}\right), \phi_{\xi}\right)$.

On the other hand, let $\mathcal{M}_{\triangleright}=\mathcal{B} \xi \oplus \mathcal{M}_{1}^{\circ} \oplus \mathcal{M}_{2}^{\circ} \oplus \mathcal{M}_{2}^{\circ} \otimes_{\mathcal{B}} \mathcal{M}_{1}^{\circ}$. Then $\mathcal{M}_{\triangleright}$ is also a $\mathcal{B}$ - $\mathcal{B}$ bimodules with specified vector such that

$$
\mathcal{M}_{\triangleright}^{\circ}=\mathcal{M}_{2}^{\circ} \oplus \mathcal{M}_{\vdash}^{\circ} \text {. }
$$

Therefore, $L\left(\mathcal{M}_{2}\right)$ and $L\left(\mathcal{M}_{\vdash}\right)$ are boolean independent in $\left(L\left(\mathcal{M}_{\triangleright}\right), \phi \xi\right)$. Let $\mathcal{J}_{2}^{\prime}=$ $\{\varnothing,(2)\}$. Then $P_{\mathcal{J}_{2}^{\prime}}^{\mathcal{M}}$ is the orthogonal projection onto $\mathcal{B} \xi \oplus \mathcal{M}_{2}^{\circ}$. It follows that the distribution of

$$
P_{\mathcal{J}_{2}^{\prime}}^{\mathcal{M}} \lambda_{2}\left(x_{2}\right)+P_{\mathcal{J}_{1}}^{\mathcal{M}} \lambda_{1}\left(x_{1}\right)+P_{\mathcal{J}_{2}}^{\mathcal{M}} \lambda_{2}\left(x_{2}\right)
$$

is the Boolean convolution of $\mu_{2}$ and $\mu_{1} \vdash \mu_{2}$. Notice that $P_{\mathcal{J}_{2}^{\prime}}^{\mathcal{M}}+P_{\mathcal{J}_{2}}^{\mathcal{M}}=P_{\mathcal{J}_{2}^{\prime \prime}}^{\mathcal{M}}$ where $\mathcal{J}_{2}^{\prime \prime}=\{\varnothing,(1),(2),(1,2)\}$, and $\mathcal{J}^{\prime}=\left\{\mathcal{J}_{1}, \mathcal{J}_{2}^{\prime \prime}\right\}$-additive convolution of $\mu_{1}$ and $\mu_{2}$ is $\mu_{1} \triangleright \mu_{2}$. Therefore, we have the following result.

Proposition 6.1. Let $\mu, \nu \in \Sigma_{0}(\mathcal{B})$. Then

$$
\mu \triangleright \nu=\nu \uplus(\mu \vdash \nu) .
$$

Proposition 6.2. Given $\mu, \nu \in \Sigma_{0}(\mathcal{B})$, for each $n \in \mathbb{N}$, we have

$$
F_{\mu \vdash \nu, n}\left(b_{n}\right)=F_{\mu, n}\left(F_{\nu, n}\left(b_{n}\right)\right)-F_{\nu, n}\left(b_{n}\right)+b_{n},
$$

for all $b_{n} \in \mathbb{H}_{n}^{+}$. 
Proof. By Proposition 6.1, Proposition 4.4 and Proposition 5.5, for each $n \in \mathbb{N}$, we have

$$
F_{\mu, n}\left(F_{\nu, n}\left(b_{n}\right)\right)=F_{\mu \vdash \nu, n}\left(b_{n}\right)+F_{\nu, n}\left(b_{n}\right)-b_{n},
$$

for all $b_{n} \in \mathbb{H}_{n}^{+}$. The proof is done.

\section{Subordination CONVOLUtion}

7.1. S-free additive convolution. In this section, we study analytic properties of the sfree additive convolutions. For $i=1,2$, let $\mu_{i}, \in \Sigma_{0}(\mathcal{B}),\left(\mathcal{M}_{i}, \xi_{i}\right)$ be a $\mathcal{B}-\mathcal{B}$ bimodules with specified vectors and $x_{i} \in\left(L\left(\mathcal{M}_{i}\right), \phi_{\xi_{i}}\right)$ such that $\mu_{i}$ is equal to the distribution of $x_{i}$. Let $(\mathcal{M}, \xi)$ be the reduced free product of $\left(\mathcal{M}_{i}, \xi_{i}\right)$ and $\lambda_{i}: L\left(\mathcal{M}_{i}\right) \rightarrow L(\mathcal{M})$ are the embedding isomorphisms defined in Section 3. Let $\left.\mathcal{J}_{1}=\left\{\left(i_{1}, \cdots, i_{m}\right) \in \mathcal{K}(\mathcal{I})\right) \mid i_{m}=1\right\} \cup\{\varnothing\}$ and $\left.\mathcal{J}_{2}=\left\{\left(i_{1}, \cdots, i_{m}\right) \in \mathcal{K}(\mathcal{I})\right) \mid i_{m}=1\right\}$. Then, $P_{\mathcal{J}_{1}}^{\mathcal{M}}$ is the orthogonal projection onto

$$
\mathcal{B} \xi \oplus \mathcal{M}_{1}^{\circ} \oplus \mathcal{M}_{2}^{\circ} \otimes_{\mathcal{B}} \mathcal{M}_{1}^{\circ} \oplus \mathcal{M}_{1}^{\circ} \otimes_{\mathcal{B}} \mathcal{M}_{2}^{\circ} \otimes_{\mathcal{B}} \mathcal{M}_{1}^{\circ} \cdots
$$

and $P_{\mathcal{J}_{2}}^{\mathcal{M}}$ is the orthogonal projection onto

$$
\mathcal{M}_{1}^{\circ} \oplus \mathcal{M}_{2}^{\circ} \otimes_{\mathcal{B}} \mathcal{M}_{1}^{\circ} \oplus \mathcal{M}_{1}^{\circ} \otimes_{\mathcal{B}} \mathcal{M}_{2}^{\circ} \otimes_{\mathcal{B}} \mathcal{M}_{1}^{\circ} \cdots
$$

Then, the s-free additive convolution $\mu_{1} \boxplus \mu_{2}$ of $\mu, \nu$ is equal to the distribution of $X=$ $P_{\mathcal{J}_{1}}^{\mathcal{M}} \lambda_{1}\left(x_{1}\right)+P_{\mathcal{J}_{2}}^{\mathcal{M}} \lambda_{2}\left(x_{2}\right)$ in $\left(L(\mathcal{M}), \phi_{\xi}\right)$.

Let $\mathcal{M}_{\square}=\mathcal{B} \xi \oplus \mathcal{M}_{b r}^{\circ}$, where

$$
\mathcal{M}_{b r}^{\circ}=\mathcal{M}_{1}^{\circ} \oplus \mathcal{M}_{2}^{\circ} \otimes \mathcal{M}_{1}^{\circ} \oplus \mathcal{M}_{1}^{\circ} \otimes \mathcal{M}_{2}^{\circ} \otimes \mathcal{M}_{1}^{\circ} \cdots
$$

Then, we have that

$$
\mathcal{M}=\mathcal{B} \xi \oplus \mathcal{M}_{b r}^{\circ} \oplus \mathcal{M}_{2}^{\circ} \oplus \mathcal{M}_{b r}^{\circ} \otimes \mathcal{M}_{2}^{\circ}
$$

and there exits a unitary $V: \mathcal{M}_{\boxplus} \otimes \mathcal{M}_{2} \rightarrow \mathcal{M}$ such that

$$
\begin{aligned}
V: & \xi \otimes_{\mathcal{B}} \xi_{2} \rightarrow \xi, \\
V: & \xi \otimes_{\mathcal{B}} m_{2} \rightarrow m_{2}, \\
V: & m_{1} \otimes_{\mathcal{B}} \xi_{2} \rightarrow m_{1}, \\
V: & m_{1} \otimes_{\mathcal{B}} m_{2} \rightarrow m_{1} \otimes_{\mathcal{B}} m_{2},
\end{aligned}
$$

where $m_{1} \in \mathcal{M}_{\square}^{\circ}, m_{2} \in \mathcal{M}_{2}^{\circ}$.

Let $\left.\lambda_{2}\left(x_{2}\right)\right|_{\mathcal{M}_{2}}$ be the restriction of $\lambda_{2}\left(x_{2}\right)$ onto $\mathcal{B} \xi \oplus \mathcal{M}_{2}^{\circ}$. Then, the distribution of $V\left(X \otimes_{\mathcal{B}} 1_{\mathcal{M}_{2}}\right) V^{-1}+\left.\lambda_{2}\left(x_{2}\right)\right|_{\mathcal{M}_{2}}$ is $\mu_{2} \triangleright \mu_{X}=\mu_{2} \triangleright\left(\mu_{1} \boxminus \mu_{2}\right)$.

\section{Lemma 7.1.}

$$
V\left(X \otimes_{\mathcal{B}} 1_{\mathcal{M}_{2}}\right) V^{-1}+\left.\lambda_{2}\left(x_{2}\right)\right|_{\mathcal{M}_{2}}=\lambda_{1}\left(x_{1}\right)+\lambda_{2}\left(x_{2}\right) .
$$


Proof. It suffices to show that

$$
\left[V\left(X \otimes_{\mathcal{B}} 1_{\mathcal{M}_{2}}\right) V^{-1}+\left.\lambda_{2}\left(x_{2}\right)\right|_{\mathcal{M}_{2}}\right](v)=\left[\lambda_{1}\left(x_{1}\right)+\lambda_{2}\left(x_{2}\right)\right](v)
$$

for all $v \in \mathcal{M}$.

We have the following cases:

Case 1: $v \in \mathcal{B} \xi$, then $v=b \xi$ for some $b \in \mathcal{B}$. We have

$$
V\left(X \otimes 1_{\mathcal{M}_{2}}\right) V^{-1} b \xi=V\left(X \otimes 1_{\mathcal{M}_{2}}\right)\left(b \xi \otimes \xi_{2}\right) .
$$

and

$$
X(b \xi)=\left(P_{\mathcal{J}_{1}}^{\mathcal{M}} \lambda_{1}\left(x_{1}\right)+P_{\mathcal{J}_{2}}^{\mathcal{M}} \lambda_{2}\left(x_{2}\right)\right)(b \xi)=\lambda_{1}\left(x_{1}\right) b \xi
$$

and

$$
\left.\lambda_{2}\left(x_{2}\right)\right|_{\mathcal{M}_{2}}(b \xi)=\lambda_{2}\left(x_{2}\right) b \xi .
$$

The equality holds in this case.

Case 2: $v \in \mathcal{M}_{2}^{\circ}$, then

$$
\begin{aligned}
V\left(X \otimes 1_{\mathcal{M}_{2}}\right) V^{-1} v & =V\left(X \otimes 1_{\mathcal{M}_{2}}\right)(\xi \otimes v) \\
& =V\left(\lambda_{1}\left(x_{1}\right) \xi \otimes v\right) \\
& =\phi_{\xi}\left(\lambda_{1}\left(x_{1}\right)\right) v+\left[\lambda_{1}\left(x_{1}\right) \xi-\phi_{\xi}\left(\lambda_{1}\left(x_{1}\right)\right)\right] \otimes v \\
& =\lambda_{1}\left(x_{1}\right) v
\end{aligned}
$$

and

$$
\left.\lambda_{2}\left(x_{2}\right)\right|_{\mathcal{M}_{2}}(v)=\lambda_{2}\left(x_{2}\right) v .
$$

The equality holds in this case.

Case 3: $v \in \mathcal{M}_{\square}^{\circ}$, then

$$
V\left(X \otimes 1_{\mathcal{M}_{2}}\right) V^{-1} v=V\left(X \otimes 1_{\mathcal{M}_{2}}\right)\left(v \otimes \xi_{2}\right)=X v
$$

and

$$
P_{\mathcal{J}_{1}}^{\mathcal{M}} v=P_{\mathcal{J}_{2}}^{\mathcal{M}} v=v
$$

Therefore, $X v=\left[\lambda_{1}\left(x_{1}\right)+\lambda_{2}(x)\right] v$. Ont the other hand, $\left.\lambda_{2}\left(x_{2}\right)\right|_{\mathcal{M}_{2}} v=0$. The equality holds in this case.

Case 3: $v \in \mathcal{M}_{\square}^{\circ} \otimes \mathcal{M}_{2}^{\circ}$, then $v=v_{1} \otimes v_{2}$ where $v_{1} \in \mathcal{M}_{\square}^{\circ}$ and $v_{2} \in \mathcal{M}_{2}^{\circ}$. Similarly, we have

$$
X v_{1}=\left[\lambda_{1}\left(x_{1}\right)+\lambda_{2}(x)\right] v_{1}
$$

and

$$
\begin{aligned}
V\left(X \otimes 1_{\mathcal{M}_{2}}\right) V^{-1} v & =V\left(X \otimes 1_{\mathcal{M}_{2}}\right)\left(v_{1} \otimes v_{2}\right) \\
& =V\left(\left[\lambda_{1}\left(x_{1}\right)+\lambda_{2}(x)\right]\left(v_{1}\right) \otimes v_{2}\right) \\
& =\left[\lambda_{1}\left(x_{1}\right)+\lambda_{2}(x)\right]\left(v_{1} \otimes v_{2}\right)
\end{aligned}
$$


and $\left.\left.\lambda_{2}\left(x_{2}\right)\right|_{\mathcal{M}_{2}}\right] v=0$. The equality holds in this case.

The proof is done.

Therefore, the distribution of $V\left(X \otimes 1_{\mathcal{M}_{2}}\right) V^{-1}+\left.\lambda_{2}\left(x_{2}\right)\right|_{\mathcal{M}_{2}}$ is $\mu_{1} \boxplus \mu_{2}$ and we have the following result.

Proposition 7.2. Let $\mu_{1}, \mu_{2} \in \Sigma_{0}(\mathcal{B})$ be two $\mathcal{B}$-valued distributions. Then, we have

$$
\mu_{2} \triangleright\left(\mu_{1} \boxplus \mu_{2}\right)=\mu_{1} \boxplus \mu_{2} .
$$

From the previous construction, we have the following generalization of Proposition 2.8 in [6].

Proposition 7.3. Let $\mu, \nu \in \Sigma_{0}(\mathcal{B})$. For each $n \in \mathbb{N}$. we have

$$
F_{\mu \boxplus \nu, n}\left(b_{n}\right)=F_{\mu, n}\left(F_{\nu \boxplus \mu, n}\left(b_{n}\right)\right)
$$

for all $b_{n} \in \mathbb{H}_{n}^{+}$.

Proof. The statement directly follows Proposition 7.2 and Proposition 5.5.

Since the $\mathcal{B}$-valued reciprocal Cauchy transform of an $\mathcal{B}$-valued distribution is one to one from a neighborhood of infinity in $\mathbb{H}^{+}$to a neighborhood of infinity in $\mathbb{H}^{+}[41], F_{\nu \boxminus \mu, n}$ is uniquely determined by

$$
F_{\mu, n}^{\langle-1\rangle}\left(F_{\mu \boxminus \nu, n}\left(b_{n}\right)\right)=F_{\mu, n}\left(F_{\nu \boxminus \mu, n}\left(b_{n}\right)\right),
$$

where $b_{n}$ is from a neighborhood of infinity in $\mathbb{H}_{n}^{+}$.

Now $\left.\widetilde{\mathcal{J}}_{1}=\left\{\left(i_{1}, \cdots, i_{m}\right) \in \mathcal{K}(\mathcal{I})\right) \mid i_{m}=2\right\}$ and $\left.\widetilde{\mathcal{J}}_{2}=\left\{\left(i_{1}, \cdots, i_{m}\right) \in \mathcal{K}(\mathcal{I})\right) \mid i_{m}=2\right\} \cup\{\varnothing\}$ Then, $P_{\widetilde{J}_{1}}^{\mathcal{M}}$ is the orthogonal projection onto

$$
\mathcal{M}_{2}^{\circ} \oplus \mathcal{M}_{1}^{\circ} \otimes_{\mathcal{B}} \mathcal{M}_{2}^{\circ} \oplus \mathcal{M}_{2}^{\circ} \otimes_{\mathcal{B}} \mathcal{M}_{1}^{\circ} \otimes_{\mathcal{B}} \mathcal{M}_{2}^{\circ} \cdots
$$

and $P_{\mathcal{J}_{2}}^{\mathcal{M}}$ is the orthogonal projection onto

$$
\mathcal{B}_{\xi} \oplus \mathcal{M}_{2}^{\circ} \oplus \mathcal{M}_{1}^{\circ} \otimes_{\mathcal{B}} \mathcal{M}_{2}^{\circ} \oplus \mathcal{M}_{2}^{\circ} \otimes_{\mathcal{B}} \mathcal{M}_{1}^{\circ} \otimes_{\mathcal{B}} \mathcal{M}_{2}^{\circ} \cdots
$$

Then, the s-free additive convolution $\mu_{2} \boxplus \mu_{1}$ of $\mu, \nu$ is equal to the distribution of $\tilde{X}=$ $P_{\widetilde{J}_{1}}^{\mathcal{M}} \lambda_{1}\left(x_{1}\right)+P_{\widetilde{\mathcal{J}}_{2}}^{\mathcal{M}} \lambda_{2}\left(x_{2}\right)$ in $\left(L(\mathcal{M}), \phi_{\xi}\right)$.

Let $\widetilde{\mathcal{M}_{\square}}=\mathcal{B} \xi \oplus \widetilde{\mathcal{M}_{b r}^{\circ}}$, where

$$
\widetilde{\mathcal{M}_{b r}^{\circ}}=\mathcal{M}_{2}^{\circ} \oplus \mathcal{M}_{1}^{\circ} \otimes_{\mathcal{B}} \mathcal{M}_{2}^{\circ} \oplus \mathcal{M}_{2}^{\circ} \otimes_{\mathcal{B}} \mathcal{M}_{1}^{\circ} \otimes_{\mathcal{B}} \mathcal{M}_{2}^{\circ} \cdots
$$

Then we have

$$
\mathcal{M}=\xi \oplus \widetilde{\mathcal{M}_{\square}^{\circ}} \oplus \mathcal{M}_{\square}^{\circ}
$$

Therefore, the distribution of $X+\tilde{X}$ is the Boolean additive convolution of $\left(\mu_{1} \boxminus \mu_{2}\right) \uplus$ $\left(\mu_{2} \boxminus \mu_{1}\right)$. Notice that

$$
X+\tilde{X}=\lambda_{1}\left(x_{1}\right)+\lambda_{2}\left(x_{2}\right)
$$


We have the following decomposition for the free additive convolutions.

Proposition 7.4. Let $\mu_{1}, \mu_{2} \in \Sigma_{0}(\mathcal{B})$ be two $\mathcal{B}$-valued distributions. Then, we have

$$
\mu_{1} \boxplus \mu_{2}=\left(\mu_{1} \boxplus \mu_{2}\right) \uplus\left(\mu_{2} \boxplus \mu_{1}\right) .
$$

Proposition 7.5. Let $\mu, \nu \in \Sigma_{0}(\mathcal{B})$. For each $n \in \mathbb{N}$, we have

$$
F_{\mu \boxplus \nu, n}\left(b_{n}\right)=F_{\nu \boxplus \mu, n}\left(b_{n}\right)+F_{\mu \boxplus \nu, n}\left(b_{n}\right)-b_{n}
$$

for all $b_{n} \in \mathbb{H}_{n}^{+}$.

Proof. The statement directly follows Proposition 7.4 and Proposition 4.4 .

7.2. S-free additive convolution and operator-valued free convolution powers. In this subsection we study relations between s-free additive convolution and the subordination functions of the operator valued free convolution powers defined in [2]. Let $\mu \in \Sigma_{0}(\mathcal{B})$ and $\alpha$ be a completely positive map such that $\eta=\alpha-1$ is also completely positive. By Theorem 8.4 in [2] and the matricial extension property of the free convolution, for each $n \in \mathbb{N}$, we have that there is a unique solution $\omega_{\alpha, \mu, n}$ to the functional equation

$$
\omega_{\alpha, \mu, n}\left(b_{n}\right)=b_{1}+\left(\alpha_{n}-1_{n}\right) h_{\mu, n}\left(\omega_{\alpha, \mu, n}\left(b_{n}\right)\right), \quad b_{n} \in \mathbb{H}_{n}^{+},
$$

where $h_{\mu, n}\left(b_{n}\right)=F_{\mu, n}\left(b_{n}\right)-b_{n}$.

Proposition 7.6. Let $\mu \in \Sigma_{0}(\mathcal{B})$. For each $n \in \mathbb{N}$, we have

$$
\omega_{\alpha, \mu, n}\left(b_{n}\right)=F_{\mu^{\uplus \eta} \amalg \mu^{\uplus \eta}}\left(b_{n}\right) .
$$

Proof. It suffices to show that $F_{\mu^{\uplus \eta} \boxplus \mu^{\uplus \eta}}$ satisfies Equation (22). By Equation (22), we have

$$
\alpha_{n}\left(\omega_{\alpha, \mu, n}\left(b_{n}\right)\right)=b_{n}+\left(\alpha_{n}-1_{n}\right) F_{\mu}\left(\omega_{\alpha, \mu, n}\left(b_{n}\right)\right), \quad b_{n} \in \mathbb{H}_{n}^{+} .
$$

By Proposition [7.5, we have

$$
2 F_{\mu^{\uplus \eta} \square \mu^{\uplus \eta}, n}\left(b_{n}\right)-b_{n}=F_{\mu^{\uplus \eta}, n}\left(F_{\mu^{\uplus \eta} \boxplus \mu^{\uplus \eta}, n}\left(b_{n}\right)\right), \quad b_{n} \in \mathbb{H}^{+} .
$$

Then, by Theorem 4.17, we have

(25) $2 F_{\mu^{\uplus \eta} \boxplus \mu^{\uplus \eta}, n}\left(b_{n}\right)-b_{n}=\eta_{n}\left[F_{\mu, n}\left(F_{\mu^{\uplus \eta} \llbracket \mu^{\uplus \eta}, n}\left(b_{n}\right)\right)\right]+\left(1_{n}-\eta_{n}\right) F_{\mu^{\uplus \eta} \boxplus \mu^{\uplus \eta}, n}\left(b_{n}\right), \quad b_{n} \in \mathbb{H}^{+}$.

Therefore, we have

$$
(1+\eta) F_{\mu^{\uplus \eta} \square \mu^{\uplus \eta}, n}\left(b_{n}\right)-b_{n}=\eta_{n}\left[F_{\mu, n}\left(F_{\mu^{\uplus \eta} \square \mu^{\uplus \eta}, n}\left(b_{n}\right)\right)\right] .
$$

Notice that $1_{n}+\eta_{n}=\alpha_{n}, F_{\mu^{\uplus \eta} \square \mu^{\uplus \eta}, n}$ is a solution to Equation 23. It follows that $\omega_{\alpha, \mu, n}=$ $F_{\mu^{\uplus \eta} \boxplus \mu^{\uplus \eta}, n}$ on $\mathbb{H}_{n}^{+}$. 


\subsection{Matricial R-transform and the free additive s-free additive convolution.}

In this subsection, we prove an operator valued version of Relations (14) and (15).

Lemma 7.7. Given $\mu, \nu \in \Sigma_{0}(\mathcal{B})$, let $\mu \boxminus \nu$ be the $s$-free additive convolution of $\mu, \nu$ and let $\left(R_{\mu, n}\right)_{n \geqslant 1}$ and $\left(R_{\mu \boxminus \nu, n}\right)_{n \geqslant 1}$ be the matricial R-transform of $\mu$ and $\mu \boxminus \nu$, respectively. Then for each $n \in \mathbb{N}$, we have that

$$
R_{\mu \llbracket \nu, n}\left(b_{n}^{-1}\right)=R_{\mu, n}\left(G_{\nu, n}\left(b_{n}\right)\right),
$$

where $b_{n}$ is from a neighborhood of infinity in $\mathbb{H}_{n}^{+}$.

Proof. Recall that $R_{\mu, n}\left(b_{n}\right)=G_{\mu, n}^{\langle-1\rangle}\left(b_{n}\right)-b_{n}^{-1}$ for $b_{n} \in \mathbb{H}_{n}^{-}$such that $\left\|b_{n}\right\|$ is small enough. Let $b_{n} \in \mathbb{H}_{n}^{+}$, then $G_{\mu \boxplus \nu}\left(b_{n}\right) \in \mathbb{H}_{n}^{-}$and we have that

$$
\begin{aligned}
R_{\mu \boxminus \nu, n}\left(G_{\mu \boxminus \nu, n}\left(b_{n}\right)\right) & =b_{n}-\left(G_{\mu \boxplus \nu, n}\left(b_{n}\right)\right)^{-1} \\
& =b_{n}-F_{\mu \boxplus \nu, n}\left(b_{n}\right) .
\end{aligned}
$$

By Proposition 7.5, if $\left\|b_{n}^{-1}\right\|$ is small enough, we have

$$
\begin{aligned}
R_{\mu \boxplus \nu, n}\left(G_{\mu \boxplus \nu, n}\left(b_{n}\right)\right) & =b_{n}-F_{\mu \boxplus \nu, n}\left(b_{n}\right) \\
& =F_{\nu \boxplus \mu, n}\left(b_{n}\right)-F_{\nu \boxplus \mu, n}\left(b_{n}\right) \\
& =F_{\mu, n}^{\langle-1\rangle}\left[F_{\nu \boxplus \mu, n}\left(b_{n}\right)\right]-F_{\nu \boxplus \mu, n}\left(b_{n}\right) .
\end{aligned}
$$

Notice that $F_{\mu, n}^{\langle-1\rangle}\left[F_{\nu \boxplus \mu, n}\left(b_{n}\right)\right]-F_{\nu \boxplus \mu, n}\left(b_{n}\right)=\phi_{\mu, n}\left(F_{\nu \boxplus \mu, n}\left(b_{n}\right)\right)$. where $\phi_{\mu, n}$ is the Voiculescu transform and $\phi_{\mu, n}(b)=R_{\mu, n}\left(b^{-1}\right)$.

It follows that

$$
R_{\mu \boxminus \nu, n}\left(G_{\mu \boxplus \nu, n}\left(b_{n}\right)\right)=R_{\mu, n}\left(G_{\mu \boxplus \nu, n}\left(b_{n}\right)\right) .
$$

By Proposition 7.3, we have that

$$
\begin{aligned}
G_{\mu \boxplus \nu, n}\left(b_{n}\right) & =\left[F_{\mu \boxplus \nu, n}\left(b_{n}\right)\right]^{-1} \\
& =\left[F_{\nu, n}\left(F_{\mu \boxplus \nu, n}\left(b_{n}\right)\right)\right]^{-1} \\
=G_{\nu, n}\left(F_{\mu \boxminus \nu, n}\left(b_{n}\right)\right) . &
\end{aligned}
$$

Therefore, we have that

$$
R_{\mu \boxminus \nu, n}\left(G_{\mu \boxplus \nu, n}\left(b_{n}\right)\right)=R_{\mu, n}\left[G_{\nu, n}\left(F_{\mu \boxplus \nu, n}\left(b_{n}\right)\right) .\right]
$$

Since the range of $G_{\mu \boxplus \nu, n}$ contains a neighborhood of 0 in $\mathbb{H}_{n}^{-}$. The proof is done.

By the linearization property of the R-transform, we have the following result. 
Proposition 7.8. Let $\mu_{1}, \mu_{2}, \mu, \nu \in \Sigma_{0}(\mathcal{B})$ and $\alpha$ is a completely positive map from $\mathcal{B}$ to B. Then

$$
\left(\mu_{1} \boxplus \mu_{2}\right) \boxplus \nu=\left(\mu_{1} \boxplus \nu\right) \boxplus\left(\mu_{2} \boxplus \nu\right) .
$$

If $\mu^{\boxplus \alpha} \in \Sigma_{0}(\mathcal{B})$, then

$$
(\mu \boxminus \nu)^{\boxplus \alpha} \in \Sigma_{0}(\mathcal{B})
$$

and

$$
(\mu \boxminus \nu)^{\boxplus \alpha}=\mu^{\boxplus \alpha} \boxplus \nu .
$$

\section{More Relations Between CONVOlutions AND transforms in OPERATOR-VALUED FREE PROBABILITY}

In this section, we use the properties in previous sections to study more relations between convolutions and transforms in operator-valued free probability. The main fact we use in the section is that the matricial reciprocal Cauchy transforms, the matricial $\mathrm{R}$ transforms, Voiculescu's transform can completely determine $\mathcal{B}$-valued distributions. We will denote by $\mathcal{C P}(\mathcal{B})$ the family of completely positive maps from $\mathcal{B}$ to $\mathcal{B}$. Given $s \in \mathcal{C P}(\mathcal{B})$, for each $n \in \mathbb{N}, s_{n}$ denotes the completely map from $\mathcal{B} \otimes M_{n}(\mathbb{C})$ to $\mathcal{B} \otimes M_{n}(\mathbb{C})$ defined as

$$
s_{n}(b \otimes e(i, j))=s(b) \otimes e,
$$

where $b \in \mathcal{B}$ and $e \in M_{n}(\mathbb{C})$.

Proposition 8.1. Let $\mu, \nu \in \Sigma_{0}(\mathcal{B})$. Then

$$
\mu \vdash(\nu \boxplus \mu)=\mu \boxminus \nu .
$$

Proof. For each $n \in \mathbb{N}, b_{n} \in \mathbb{H}_{n}^{+}$, by Proposition 6.2, 7.3.7.5, we have

$$
\begin{aligned}
F_{\mu \vdash(\nu \boxplus \mu), n}\left(b_{n}\right) & =F_{\mu, n}\left(F_{\nu \boxplus \mu, n}\left(b_{n}\right)\right)-F_{\nu \boxplus \mu, n}\left(b_{n}\right)+b_{n} \\
& =F_{\nu \boxplus \mu, n}\left(b_{n}\right)-F_{\nu \boxplus \mu, n}\left(b_{n}\right)+b_{n} \\
& =F_{\mu \boxplus \nu, n}\left(b_{n}\right) .
\end{aligned}
$$

The proof is done.

Recall that a $\mathcal{B}$-valued Boolean central limit law or say $\mathcal{B}$-valued Bernoulli law is completely determine by its variance which is a completely positive map $s \in \mathcal{C P}(\mathcal{B})$ [5]. If we denote by $\operatorname{Ber}_{s}$ be the $\mathcal{B}$-valued Bernoulli law of variance $s$, then

$$
F_{\text {Ber }, n_{s}}\left(b_{n}\right)=b_{n}-s_{n}\left(b_{n}^{-1}\right),
$$

for $b_{n} \in \mathbb{H}_{n}^{+}$. We will write Ber short for $B e r_{1}$. 
On the other hand, a $\mathcal{B}$-valued free central limit law or say $\mathcal{B}$-valued semicircularlaw is completely determine by its variance which is a completely positive map $s \in \mathcal{C P}(\mathcal{B})$ [5]. If we denote by $\gamma_{s}$ the $\mathcal{B}$-valued semicircular law of variance $s$, then the matricial Voiculescu's transform $\left(\phi_{\gamma, n}\right)$ are given by the following formula

$$
\phi_{\gamma, n}\left(b_{n}\right)=s_{n}\left(b_{n}^{-1}\right)
$$

for $b_{n} \in \mathbb{H}_{n}^{+}$in a neighborhood of infinity. We will write $\gamma$ short for $\gamma_{1}$.

Proposition 8.2. Given $s \in \mathcal{C P}(\mathcal{B})$, we have

$$
\operatorname{Ber}_{s} \vdash \gamma_{s}=\gamma_{s} .
$$

Proof. By Proposition 6.2, for $n \in \mathbb{N}$, we have that

$$
\begin{aligned}
F_{B e r_{s} \vdash \gamma_{s}, n}\left(b_{n}\right) & =F_{B e r_{s}, n}\left(F_{\gamma_{s}, n}\left(b_{n}\right)\right)-F_{\gamma_{s}, n}\left(b_{n}\right)+b_{n} \\
& =F_{\gamma_{s}, n}\left(b_{n}\right)-s_{n}\left(G_{\gamma_{s}, n}\left(b_{n}\right)\right)-F_{\gamma_{s}, n}\left(b_{n}\right)+b_{n} \\
& \left.=F_{\gamma_{s}, n}\left(b_{n}\right)-\phi_{\gamma_{s}, n}\left(F_{\gamma_{s}, n}\left(b_{n}\right)\right)\right)-F_{\gamma_{s}, n}\left(b_{n}\right)+b_{n} .
\end{aligned}
$$

According to the definition of the matricial Voiculescu transform, we have that

$$
\phi_{\mu, n}\left(b_{n}\right)=F_{\mu, n}^{\langle-1\rangle}\left(b_{n}\right)-b_{n} .
$$

Replace $b_{n}$ by $F_{\mu, n}\left(b_{n}\right)$, then we have we have

$$
\phi_{\mu, n}\left(F_{\mu, n}\left(b_{n}\right)\right)=b_{n}-F_{\mu, n}\left(b_{n}\right) .
$$

Therefore, we have

$$
F_{B e r_{s} \vdash \gamma_{s}, n}\left(b_{n}\right)=F_{\gamma_{s}, n}\left(b_{n}\right) .
$$

An operator valued Belinschi-Nica transform $\Phi$ is defined combinatorially in [2]. It is not clear that if the transform is a map from $\Sigma_{0}(\mathcal{B})$ to $\Sigma_{0}(\mathcal{B})$. Below, we give an equivalent definition of $\Phi$ by a map from $\Sigma_{0}(\mathcal{B})$ to $\Sigma_{0}(\mathcal{B})$ which is an operator-valued analogue of relation (12).

Definition 8.3. Let $\mu \in \Sigma_{0}(\mathcal{B})$. We define $\Phi: \Sigma_{0}(\mathcal{B}) \rightarrow \Sigma_{0}(\mathcal{B})$ such that

$$
\Phi(\mu)=\operatorname{Ber} \vdash \mu,
$$

for $\mu \in \Sigma_{0}(\mathcal{B})$. Then, for each $n \in \mathbb{N}$, we have that

$$
F_{\Phi(\mu), n}\left(b_{n}\right)=b_{n}-G_{\mu, n}\left(b_{n}\right),
$$

for $b_{n} \in \mathbb{H}_{n}^{+}$.

The following proposition exhibits a relation between the standard semicircular law, $\Phi$-transform and the s-free additive convolution. 
Proposition 8.4. Let $\mu \in \Sigma_{0}(\mathcal{B})$. Then we have

$$
\gamma \boxplus \mu=\Phi(\gamma \boxplus \mu) .
$$

Proof. For each $n \in \mathbb{N}, b_{n} \in \mathbb{H}_{n}^{+}$, we have

$$
\begin{aligned}
F_{\gamma \boxplus \mu, n}\left(b_{n}\right) & =F_{\gamma \boxplus \mu, n}\left(b_{n}\right)-F_{\mu \boxplus \gamma, n}\left(b_{n}\right)+b_{n} \\
& =F_{\gamma \boxplus \mu, n}\left(b_{n}\right)+G_{\gamma \boxplus \mu},\left(b_{n}\right)-F_{\mu \boxplus \gamma,}\left(b_{n}\right)-G_{\gamma \boxplus \mu},\left(b_{n}\right)+b_{n} \\
& =F_{\gamma, n}^{<-1>}\left(F_{\gamma \boxplus \mu, n}\left(b_{n}\right)\right)-F_{\mu \boxplus \gamma, n}\left(b_{n}\right)-G_{\gamma \boxplus \mu, n}\left(b_{n}\right)+b_{n} \\
& =-G_{\gamma \boxplus \mu, n}\left(b_{n}\right)+b_{n} \\
& =F_{\Phi(\gamma \boxplus \mu), n}\left(b_{n}\right) .
\end{aligned}
$$

The proof is done.

Now we turn to study the $\mathcal{B}$-valued $\mathbb{B}$-transform which is already given in $[2]$.

Definition 8.5. Let $s \in \mathcal{C P}(\mathcal{B})$. The map $\mathbb{B}_{s}: \Sigma_{0}(\mathcal{B}) \rightarrow \Sigma_{0}(\mathcal{B})$ is defined by the following formula

$$
\mathbb{B}_{s}(\mu)=\left(\mu^{\boxplus(1+s)}\right)^{\uplus(1+s)^{-1}},
$$

for $\mu \in \Sigma_{0}(\mathcal{B})$.

The following proposition is proven combinatorially in [2].

Proposition 8.6. Given $s, t \in \mathcal{C} \mathcal{P}(\mathcal{B})$, then we have

$$
\mathbb{B}_{s}\left(\mathbb{B}_{t}(\mu)\right)=\mathbb{B}_{s+t}(\mu),
$$

for all $\mu \in \Sigma_{0}(\mathcal{B})$.

One would see that this proposition can be derived from the following result which is a generalization of Relation (11).

Proposition 8.7. Let $p, q \in \mathcal{C P}(\mathcal{B})$ such that $p-1$ and $1-p+q p$ are invertible completely positive maps. Then we have that

$$
\left(\mu^{\boxplus p}\right)^{\uplus q}=\left(\mu^{\uplus q^{\prime}}\right)^{\boxplus p^{\prime}},
$$

where $p^{\prime}, q^{\prime} \in \mathcal{C P}(\mathcal{B})$ are defined as follows:

$$
p^{\prime}=q p(1-p+q p)^{-1}, \quad q^{\prime}=1-p+q p .
$$

Proof. We just need to compare the matricial reciprocal Cauchy transforms of the two distributions. By Proposition 4.17, for each $n \in \mathbb{N}$ and $b_{n} \in \mathbb{H}_{n}^{+}$, we have

$$
F_{(\mu \boxplus p) \uplus q, n}\left(b_{n}\right)=q_{n}\left[F_{\mu \boxplus p, n}\left(b_{n}\right)\right]+\left(1_{n}-q_{n}\right) b_{n} .
$$


By Proposition 7.6, and equation (23), we have that

$$
F_{\mu^{\boxplus p}, n}\left(b_{n}\right)=\left(p_{n}-1_{n}\right)^{-1} p_{n} F_{\mu^{\uplus(p-1)} \boxplus \mu^{\uplus(p-1)}, n}\left(b_{n}\right)-\left(p_{n}-1_{n}\right)^{-1} b_{n} .
$$

Therefore, we have that

$$
F_{\left(\mu^{\boxplus p}\right) \uplus q, n}\left(b_{n}\right)=q_{n}\left(p_{n}-1+n\right)^{-1} p_{n} F_{\mu^{\uplus(p-1)} \boxplus \mu^{\uplus(p-1)}, n}\left(b_{n}\right)+\left(1_{n}-q_{n}\left(p_{n}-1\right)^{-1} p_{n}\right) b_{n} .
$$

On the other hand, apply Proposition 7.6 to $F_{\left(\mu^{\uplus} q^{\prime}\right) \boxplus p^{\prime}, n}\left(b_{n}\right)$ we have that

$$
F_{\left(\mu^{\uplus q^{\prime}}\right) \boxplus p^{\prime}}\left(b_{n}\right)=\left(p_{n}^{\prime}-1_{n}\right)^{-1} t \omega_{q^{\prime}, p^{\prime}}\left(b_{n}\right)+\left(1_{n}-\left(p_{n}^{\prime}-1\right)^{-1} q_{n}^{\prime}\right) b_{n},
$$

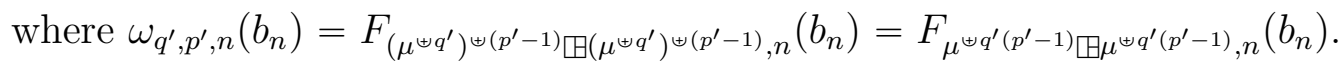

Let $q^{\prime}=1-p+q p$ and $p^{\prime}=q p(1-p+q p)^{-1}$. Then we have

$$
q^{\prime}\left(p^{\prime}-1\right)=p-1
$$

and

$$
\left(p^{\prime}-1\right)^{-1} t=q(p-1)^{-1}
$$

In this case, we have that

$$
F_{\left(\mu^{\uplus q^{\prime}}\right) \boxplus p^{\prime}}\left(b_{n}\right)=F_{\left(\mu^{\uplus} q^{\prime}\right) \boxplus p^{\prime}}\left(b_{n}\right) .
$$

The proof is done.

The following is a proof of Proposition 8.6 by use the Proposition 8.7 .

Proof. Apply Proposition 8.7 by setting $p=(s+1)(s+t+1)^{-1}$ and $q=(s+t+1)(t+1)^{-1}$, then $p^{\prime}=s+1$ and $q^{\prime}=(1+t)^{-1}$. Therefore, we have

$$
\left(\mu^{\boxplus(s+1)(s+t+1)^{-1}}\right)^{\uplus(s+t+1)(t+1)^{-1}}=\left(\mu^{\uplus(1+t)^{-1}}\right)^{\boxplus s+1} .
$$

It follows that

$$
\begin{aligned}
\mathbb{B}_{s}\left(\mathbb{B}_{t}(\mu)\right) & =\left(\left(\left(\mu^{\boxplus 1+t}\right)^{\uplus(1+t)^{-1}}\right)^{\boxplus 1+s}\right)^{\uplus(1+s)^{-1}} \\
& =\left(\left(\left(\mu^{\boxplus 1+t}\right)^{\boxplus(1+t+s)(1+t)^{-1}}\right)^{\uplus(1+s)(1+t+s)^{-1}}\right)^{\uplus(1+s)^{-1}} \\
& =\left(\mu^{\boxplus 1+t+s}\right)^{\uplus(1+t+s)^{-1}} .
\end{aligned}
$$

The proof is done.

We provide one more application of Proposition 8.7 in studying the $\mathbb{B}$-transforms. Given $s \in \mathcal{C P}(\mathcal{B})$ such that $s \geqslant 1$ and $\mu \in \Sigma_{0}(\mathcal{B})$, we have that $\mu^{\boxplus s} \in \Sigma_{0}(\mathcal{B})$ [2, 26]. By Theorem 8.4 in [2] and Proposition 4.17, we have that $\mu^{\uplus(s-1)} \boxminus \mu^{\uplus(s-1)}$ is the distribution subordinate to $\mu^{\boxplus s}$ with respect to $\mu$, namely

$$
F_{\mu, n}\left(F_{\mu^{\uplus(s-1)} \boxminus \mu^{\uplus(s-1)}}\right)=F_{\mu^{\boxplus s}, n},
$$

for all $n$. The following result is an operator valued generalization of Proposition 1.12 in [21]. 
Proposition 8.8. Given $s \in \mathcal{C P}(\mathcal{B})$ and $\mu \Sigma_{0}(\mathcal{B})$, then we have

$$
\mu^{\uplus s} \boxminus \mu^{\uplus s}=(\mathbb{B}(\mu))^{\boxplus s} .
$$

Proof. By Proposition 7.4, we have that

$$
\begin{aligned}
\mu^{\uplus s} \boxplus \mu^{\uplus s} & =\left[\mu^{\uplus s} \boxplus \mu^{\uplus s}\right] \uplus\left[\mu^{\uplus s} \boxplus \mu^{\uplus s}\right] \\
& =\left[\mu^{\uplus s} \boxplus \mu^{\uplus s}\right]^{\uplus 2} .
\end{aligned}
$$

Therefore, we have

$$
\mu^{\uplus s} \boxplus \mu^{\uplus s}=\left[\mu^{\uplus s} \boxplus \mu^{\uplus s}\right]^{\uplus 1 / 2} .
$$

Let $p=1+s$ and $q=2 s(1+s)^{-1}$. Then the $p^{\prime}, q^{\prime}$ in Proposition 8.7 are

$$
p^{\prime}=2, \quad q^{\prime}=s .
$$

It follows that

$$
\begin{aligned}
\left(\left(\mu^{\uplus s}\right)^{\boxplus 2}\right)^{\uplus 1 / 2} & =\left(\left(\mu^{\boxplus 1+s}\right)^{\boxplus 2 s(1+s)}\right)^{\uplus 1 / 2} \\
& =\left(\mu^{\boxplus 1+s}\right)^{\boxplus s(1+s)} .
\end{aligned}
$$

On the other hand, let $p=(1+s) / 2$ and $q=s(1+s)^{-1}$. Then the $p^{\prime}, q^{\prime}$ in Proposition 8.7 are

$$
\begin{aligned}
p^{\prime} & =s, \quad q^{\prime}=1 / 2 . \\
(\mathbb{B}(\mu))^{\boxplus s} & =\left(\left(\mu^{\boxplus 2}\right)^{\uplus 1 / 2}\right)^{\uplus s} \\
& =\left(\left(\mu^{\boxplus 2}\right)^{\boxplus(1+s) / 2}\right)^{\uplus s(1+s)^{-1}} \\
& =\left(\mu^{\boxplus 1+s}\right)^{\boxplus s(1+s)} .
\end{aligned}
$$

The proof is done.

Definition 8.9. Let $\mu \in \Sigma_{0}(\mathcal{B}) . \mu$ is $\boxplus$-infinitely divisible if $\mu^{\boxplus 1 / n} \in \Sigma_{0}(\mathcal{B})$ for all $n \geqslant 1$.

We see that in Proposition 8.8, we actually proved the the following relation

$$
\mathbb{B}\left(\mu^{\uplus s}\right)=\mathbb{B}(\mu)^{\boxplus s} .
$$

Let $s \in \mathcal{C P}(\mathcal{B})$ be invertible. Then we have

$$
\mathbb{B}(\mu)=\left[\mathbb{B}\left(\mu^{\uplus s^{-1}}\right)\right]^{\boxplus s} .
$$

Notice that $\mu^{\uplus s^{-1}} \in \Sigma_{0}(\mathcal{B})$ if $\nu \in \Sigma_{0}(\mathcal{B})$. Therefore, $\mathbb{B}(\mu)$ is $\boxplus$-infinitely divisible for all $\mu \in \Sigma_{0}(\mathcal{B})$ which is the property of the scalar Bercovici-Pata bijection.

Now, we turn to study an operator valued generalization of Anshelevich's free convolution with two states. 
Lemma 8.10. Let $\mu \in \Sigma_{0}(\mathcal{B})$ and $s$ is a completely positive map from $\mathcal{B}$ to $\mathcal{B}$ such $\mu^{\boxplus s} \in \Sigma_{0}(\mathcal{B})$. Then, for each $\left.n\right] \in \mathbb{N}$, we have that

$$
F_{\mu \boxplus s, n}^{\langle-1\rangle}\left(b_{n}\right)=s_{n}\left[F_{\mu, n}^{\langle-1\rangle}\left(b_{n}\right)\right]+\left(1_{n}-s_{n}\right) b_{n},
$$

$b_{n} \in \mathbb{H}_{n}^{+}$is a in sufficiently small neighborhood of infinity.

Proof. For each $n \in \mathbb{N}$, we have that

$$
\phi_{\mu, n}\left(b_{n}\right)=F_{\mu, n}^{\langle-1\rangle}\left(b_{n}\right)-b_{n} .
$$

and

$$
\phi_{\mu \boxplus s, n}\left(b_{n}\right)=F_{\mu^{\boxplus s}, n}^{\langle-1\rangle}\left(b_{n}\right)-b_{n} .
$$

Notice that

$$
\phi_{\mu \boxplus s, n}\left(b_{n}\right)=s_{n}\left[\phi_{\mu, n}\left(b_{n}\right)\right] .
$$

The result follows by simplifying the above equation.

Proposition 8.11. Given $\mu, \nu \in \Sigma_{0}(\mathcal{B})$ and $s \in \mathcal{C P}(\mathcal{B})$ such that $\mu^{\boxplus s} \in \Sigma_{0}(\mathcal{B})$, then we have

$$
\mathbb{B}_{s}(\mu \boxminus \nu)=\mu \boxminus\left(\mu^{\boxplus s} \boxplus \nu\right) .
$$

Proof. For each $n \in \mathbb{N}, b_{n} \in \mathbb{H}_{n}^{+}$, by Proposition 7.5, we have

$$
\begin{aligned}
& F_{\mu \boxplus\left(\mu^{\boxplus s} \boxplus \nu\right), n}\left(b_{n}\right)=F_{\mu \boxplus\left(\mu^{\boxplus s} \boxplus \nu\right), n}\left(b_{n}\right)-F_{(\mu \boxplus s \boxplus \nu) \boxplus, n}\left(b_{n}\right)+b_{n} \\
& =F_{\mu \boxplus(s+1) \boxplus \nu, n}\left(b_{n}\right)-F_{\left(\mu^{\boxplus s} \boxplus \nu\right) \boxplus \mu, n}\left(b_{n}\right)+b_{n}
\end{aligned}
$$

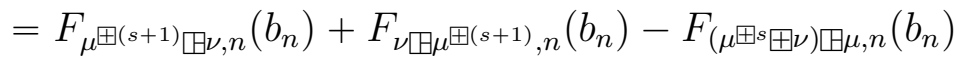

$$
\begin{aligned}
& =F_{\mu^{\boxplus(s+1)} \boxplus \nu, n}\left(b_{n}\right)+F_{\nu \boxplus \mu \boxplus(s+1), n}\left(b_{n}\right)-F_{\mu, n}^{\langle-1\rangle}\left[F_{\mu \boxplus(s+1) \boxplus \nu, n}\left(b_{n}\right)\right] .
\end{aligned}
$$

Notice that $1+s$ is invertible, by Lemma 8.10, we have that

$$
F_{\mu^{\boxplus(1+s)}, n}^{\langle-1\rangle}\left(b_{n}\right)=\left(1+s_{n}\right)\left[F_{\mu, n}^{\langle-1\rangle}\left(b_{n}\right)\right]-s_{n} b_{n} .
$$

Therefore, we have

$$
\left(1+s_{n}\right)^{-1}\left[F_{\mu^{\boxplus(1+s)}, n}^{\langle-1\rangle}\left(b_{n}\right)+s_{n} b_{n}\right]=F_{\mu, n}^{\langle-1\rangle}\left(b_{n}\right) .
$$

It follows that

$$
\begin{aligned}
& F_{\mu, n}^{\langle-1\rangle}\left[F_{\mu \boxplus(s+1) \boxplus \nu, n}\left(b_{n}\right)\right] \\
= & \left(1+s_{n}\right)^{-1}\left[F_{\mu^{\boxplus(1+s)}, n}^{\langle-1\rangle}\left(F_{\mu \boxplus(s+1) \boxplus \nu, n}\left(b_{n}\right)\right)+s_{n} F_{\mu \boxplus(s+1) \boxplus \nu, n}\left(b_{n}\right)\right] \\
= & \left(1+s_{n}\right)^{-1}\left[F_{\mu \boxplus(1+s), n}^{\langle-1\rangle}\left(F_{\mu^{\boxplus(s+1)} \boxplus \nu, n}\left(b_{n}\right)\right)\right]+\left(1+s_{n}\right)^{-1} s_{n}\left[F_{\mu \boxplus(s+1) \boxplus \nu, n}\left(b_{n}\right)\right] \\
= & \left(1+s_{n}\right)^{-1}\left[F_{\nu \boxplus \mu \boxplus(s+1), n}\left(b_{n}\right)\right]+\left(1+s_{n}\right)^{-1} s_{n}\left[F_{\mu \boxplus(s+1) \boxplus \nu, n}\left(b_{n}\right)\right] .
\end{aligned}
$$


Then, the Equation (28) becomes

$$
\begin{aligned}
& F_{\mu \boxplus(\mu \boxplus s \boxplus \nu), n}\left(b_{n}\right) \\
& =F_{\mu \boxplus(s+1) \boxplus \nu, n}\left(b_{n}\right)+F_{\nu \boxplus \mu \boxplus(s+1), n}\left(b_{n}\right)-\left\{\left(1+s_{n}\right)^{-1}\left[F_{\nu \boxplus \mu \boxplus(s+1), n}\left(b_{n}\right)\right]+\left(1+s_{n}\right)^{-1} s_{n}\left[F_{\mu \boxplus(s+1) \boxplus \nu, n}\left(b_{n}\right)\right]\right\} \\
& =F_{\mu \boxplus(s+1) \boxplus \nu, n}\left(b_{n}\right)+\left(1+s_{n}\right)^{-1} s_{n}\left[F_{\nu \boxplus \mu \boxplus(s+1), n}\left(b_{n}\right)\right]-\left(1+s_{n}\right)^{-1} s_{n}\left[F_{\mu \boxplus(s+1) \boxplus \nu, n}\left(b_{n}\right)\right] \\
& =F_{\mu^{\boxplus(s+1)} \boxplus \nu, n}\left(b_{n}\right)+\left(1+s_{n}\right)^{-1} s_{n}\left[F_{\nu \boxplus \mu \boxplus(s+1), n}\left(b_{n}\right)-F_{\mu \boxplus(s+1) \boxplus \nu, n}\left(b_{n}\right)\right]
\end{aligned}
$$

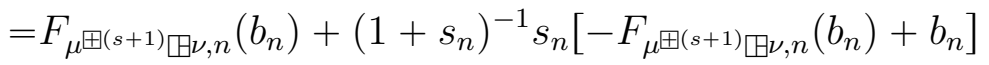

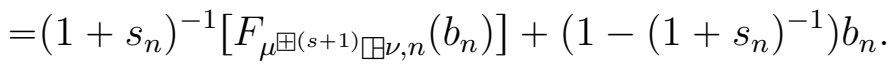

By Proposition 4.17, we have

$$
F_{\mu \boxplus\left(\mu^{\boxplus s} \boxplus \nu\right), n}\left(b_{n}\right)=F_{\left(\mu^{\boxplus(s+1)} \boxplus \nu\right)^{\uplus(1+s)^{-1}}, n}\left(b_{n}\right) .
$$

Therefore,

$$
\mu \boxminus\left(\mu^{\boxplus s} \boxplus \nu\right)=\left(\mu^{\boxplus(s+1)} \boxminus \nu\right)^{\uplus(1+s)^{-1}} .
$$

By Proposition 7.8, we have

$$
\left(\mu^{\boxplus(s+1)} \boxminus \nu\right)^{\uplus(1+s)^{-1}}=\left((\mu \boxminus \nu)^{\boxplus(s+1)}\right)^{\uplus(1+s)^{-1}}=\mathbb{B}_{s}(\mu \boxminus \nu) .
$$

The proof is done.

Corollary 8.12. Let $\nu \in \Sigma_{0}(\mathcal{B}), s \in \mathcal{C P}(\mathcal{B})$ and $\gamma_{s}$ be the semicircular of variance $s$. Then

$$
\Phi\left(\gamma_{s} \boxplus \nu\right)=\mathbb{B}_{s}(\Phi(\nu))
$$

Proof. The statement follows Proposition 8.4 and Proposition 8.11 by letting $\mu=\gamma$.

\section{REFERENCES}

[1] Michael Anshelevich. Two-state free Brownian motions. J. Funct. Anal., 260(2):541-565, 2011.

[2] Michael Anshelevich, Serban T. Belinschi, Maxime Fevrier, and Alexandru Nica. Convolution powers in the operator-valued framework. Trans. Amer. Math. Soc., 365(4):2063-2097, 2013.

[3] S. T. Belinschi and H. Bercovici. Atoms and regularity for measures in a partially defined free convolution semigroup. Math. Z., 248(4):665-674, 2004.

[4] S. T. Belinschi and H. Bercovici. A new approach to subordination results in free probability. $J$. Anal. Math., 101:357-365, 2007.

[5] S. T. Belinschi, M. Popa, and V. Vinnikov. Infinite divisibility and a non-commutative Boolean-tofree Bercovici-Pata bijection. J. Funct. Anal., 262(1):94-123, 2012.

[6] Serban T. Belinschi, Tobias Mai, and Roland Speicher. Analytic subordination theory of operatorvalued free additive convolution and the solution of a general random matrix problem. J. Reine Angew. Math., 732:21-53, 2017.

[7] Serban T. Belinschi and Alexandru Nica. On a remarkable semigroup of homomorphisms with respect to free multiplicative convolution. Indiana Univ. Math. J., 57(4):1679-1713, 2008. 
[8] Serban T. Belinschi and Alexandru Nica. Free Brownian motion and evolution towards $\boxplus$-infinite divisibility for $k$-tuples. Internat. J. Math., 20(3):309-338, 2009.

[9] Serban T. Belinschi, Roland Speicher, John Treilhard, and Carlos Vargas. Operator-valued free multiplicative convolution: analytic subordination theory and applications to random matrix theory. Int. Math. Res. Not. IMRN, (14):5933-5958, 2015.

[10] Serban Teodor Belinschi. Complex analysis methods in noncommutative probability. ProQuest LLC, Ann Arbor, MI, 2005. Thesis (Ph.D.)-Indiana University.

[11] Hari Bercovici and Vittorino Pata. Stable laws and domains of attraction in free probability theory. Ann. of Math. (2), 149(3):1023-1060, 1999. With an appendix by Philippe Biane.

[12] Hari Bercovici and Dan Voiculescu. Free convolution of measures with unbounded support. Indiana Univ. Math. J., 42(3):733-773, 1993.

[13] Philippe Biane. Processes with free increments. Math. Z., 227(1):143-174, 1998.

[14] Gennadii P. Chistyakov and Friedrich Götze. The arithmetic of distributions in free probability theory. Cent. Eur. J. Math., 9(5):997-1050, 2011.

[15] Kenneth J. Dykema. Exactness of reduced amalgamated free product $C^{*}$-algebras. Forum Math., 16(2):161-180, 2004.

[16] Uwe Franz. Monotone and Boolean convolutions for non-compactly supported probability measures. Indiana Univ. Math. J., 58(3):1151-1185, 2009.

[17] Dmitry S. Kaliuzhnyi-Verbovetskyi and Victor Vinnikov. Foundations of free noncommutative function theory, volume 199 of Mathematical Surveys and Monographs. American Mathematical Society, Providence, RI, 2014.

[18] E. C. Lance. Hilbert $C^{*}$-modules, volume 210 of London Mathematical Society Lecture Note Series. Cambridge University Press, Cambridge, 1995. A toolkit for operator algebraists.

[19] Romuald Lenczewski. Decompositions of the free additive convolution. J. Funct. Anal., 246(2):330$365,2007$.

[20] Naofumi Muraki. Monotonic independence, monotonic central limit theorem and monotonic law of small numbers. Infin. Dimens. Anal. Quantum Probab. Relat. Top., 4(1):39-58, 2001.

[21] Alexandru Nica. Multi-variable subordination distributions for free additive convolution. J. Funct. Anal., 257(2):428-463, 2009.

[22] Alexandru Nica and Roland Speicher. Lectures on the combinatorics of free probability, volume 335 of London Mathematical Society Lecture Note Series. Cambridge University Press, Cambridge, 2006.

[23] Mihai Popa. A combinatorial approach to monotonic independence over a $C^{*}$-algebra. Pacific J. Math., 237(2):299-325, 2008.

[24] Mihai Popa. A new proof for the multiplicative property of the Boolean cumulants with applications to the operator-valued case. Colloq. Math., 117(1):81-93, 2009.

[25] Mihai Popa and Victor Vinnikov. Non-commutative functions and the non-commutative free LévyHinčin formula. Adv. Math., 236:131-157, 2013.

[26] D. Shlyakhtenko. On operator-valued free convolution powers. Indiana Univ. Math. J., 62(1):91-97, 2013.

[27] Roland Speicher. On universal products. In Free probability theory (Waterloo, ON, 1995), volume 12 of Fields Inst. Commun., pages 257-266. Amer. Math. Soc., Providence, RI, 1997.

[28] Roland Speicher. Combinatorial theory of the free product with amalgamation and operator-valued free probability theory. Mem. Amer. Math. Soc., 132(627):x+88, 1998. 
[29] Roland Speicher and Reza Woroudi. Boolean convolution. In Free probability theory (Waterloo, ON, 1995), volume 12 of Fields Inst. Commun., pages 267-279. Amer. Math. Soc., Providence, RI, 1997.

[30] Jun Tomiyama. On the projection of norm one in $W^{*}$-algebras. Proc. Japan Acad., 33:608-612, 1957.

[31] D. V. Voiculescu, K. J. Dykema, and A. Nica. Free random variables, volume 1 of CRM Monograph Series. American Mathematical Society, Providence, RI, 1992. A noncommutative probability approach to free products with applications to random matrices, operator algebras and harmonic analysis on free groups.

[32] Dan Voiculescu. Symmetries of some reduced free product $C^{*}$-algebras. In Operator algebras and their connections with topology and ergodic theory (Buşteni, 1983), volume 1132 of Lecture Notes in Math., pages 556-588. Springer, Berlin, 1985.

[33] Dan Voiculescu. Addition of certain noncommuting random variables. J. Funct. Anal., 66(3):323-346, 1986.

[34] Dan Voiculescu. The analogues of entropy and of Fisher's information measure in free probability theory. I. Comm. Math. Phys., 155(1):71-92, 1993.

[35] Dan Voiculescu. Operations on certain non-commutative operator-valued random variables. Astérisque, (232):243-275, 1995. Recent advances in operator algebras (Orléans, 1992).

[36] Dan Voiculescu. The coalgebra of the free difference quotient and free probability. Internat. Math. Res. Notices, (2):79-106, 2000.

[37] Dan Voiculescu. Analytic subordination consequences of free Markovianity. Indiana Univ. Math. J., 51(5):1161-1166, 2002.

[38] Dan Voiculescu. Free analysis questions. I. Duality transform for the coalgebra of $\partial_{X}: B$. Int. Math. Res. Not., (16):793-822, 2004.

[39] Dan-Virgil Voiculescu. Free analysis questions II: the Grassmannian completion and the series expansions at the origin. J. Reine Angew. Math., 645:155-236, 2010.

[40] Dan-Virgil Voiculescu. Free probability for pairs of faces I. Comm. Math. Phys., 332(3):955-980, 2014.

[41] John D. Williams. Analytic function theory for operator-valued free probability. J. Reine Angew. Math., 729:119-149, 2017.

Department of Mathematics

Indiana University

Bloomington, IN 47401, USA

E-MAIL: liuweih@indiana.edu 